\title{
EL LUGAR DEL ISQLĀH AL-MA ŶISTTI DE ŶĀBIR B. AFLAḤ EN LA LLAMADA «REBELIÓN ANDALUSÍ CONTRA LA ASTRONOMÍA PTOLEMAICA» ${ }^{1}$
}

\author{
THE ROLE OF JĀBIR B. AFLAḤ'S ISSLĀH AL-MAJISṬI IN \\ THE SO CALLED «ANDALUSIAN REVOLT AGAINST \\ PTOLEMAIC ASTRONOMY»
}

\author{
José BELLVER MARTÍNEZ \\ Universidad de Barcelona
}

\begin{abstract}
Ŷābir b. Aflah al-Išbīlī, conocido como Geber filius Afflay Hispalensis en la Europa medieval, fue un matemático y astrónomo teórico que probablemente floreció en Sevilla durante el primer cuarto del s. XII. Ŷābir b. Aflah es un astrónomo medieval importante gracias a su obra principal, Ișlāh al-Maŷisțī, traducida al latín y al hebreo. Con el Iṣlāh al-Maŷisțì, su autor pretende reescribir el Almagesto a la vez que introduce algunas correcciones. En 1984, A.I. Sabra, en su importante artículo «The Andalusian revolt against Ptolemaic astronomy» llama la atención sobre un grupo de filósofos andalusíes, contemporáneos de Ŷābir b. Aflah o que florecieron poco después que él —como Ibn Bāŷŷa (m. 537/1138), Ibn Ṭufayl (m. 581/1185), Ibn Rušd (m. 595/1198), Maimónides (m. 601/1204) y al-Biṭrūŷī (fl. 596/1200) - , quienes criticaron, desde un punto de vista cosmológico, los modelos astronómicos descritos en el Almagesto al no ser coherentes con la física aristotélica. La finalidad de este artículo es describir el tipo de críticas que hace Ŷābir b. Aflah a Ptolomeo con el objeto de dilucidar si cabe considerarlo uno de los miembros de la rebelión andalusí contra la astronomía ptolemaica. La con-
\end{abstract}

Jābir b. Aflah al-Ishbīlī, known as Geber filius Afflay Hispalensis in medieval Western Europe, was a mathematician and theoretical astronomer who most probably flourished in Seville during the first quarter of the $12^{\text {th }}$ century. Jābir b. Aflah was a leading figure in medieval astronomy thanks to the Ișlāh al-Majisți, his magnum opus, which was translated into Latin and Hebrew. With his Iṣlāh al-Majisțī, Jābir b. Aflah intended to rewrite the Almagest and introduced some corrections to it. In his important paper «The Andalusian revolt against Ptolemaic astronomy» published in 1984, the renowned scholar A.I. Sabra drew attention to a group of Andalusian philosophers who where contemporaries of Jābir b. Aflah or flourished shortly after him, such as Ibn Bājja (d. 537/1138), Ibn Țufayl (d. 581/1185), Ibn Rushd (d. 595/1198), Maimonides (d. 601/1204) and al-Bitrūjīi ( $f$ l. 596/1200). Sabra stressed their cosmological criticisms of the astronomical models described in the Almagest arising from the lack of consistency of Ptolemy's models with Aristotle's physics. The purpose of this paper is to describe Jābir b. Aflah's criticisms of Ptolemy in order to consider whether he should be

${ }^{1}$ Este artículo ha sido redactado dentro del programa de investigación "La evolución de la ciencia en la sociedad de al-Andalus desde la Alta Edad Media al pre-Renacimiento y su repercusión en las culturas europeas y árabes (siglos X-XV)", subvencionado por el Ministerio de Educación y Ciencia (FFI2008-00234/FILO) y FEDER. 
clusión final es que las críticas de Ŷābir b. Aflah no son de carácter cosmológico - si bien puedan tener consecuencias cosmológicas-, sino que son de carácter matemático y técnico. Así pues, no cabe considerarlo un miembro de la rebelión andalusí contra la astronomía ptolemaica.

Palabras clave: Astronomía; Astronomía griega; Astronomía medieval; Astronomía islámica; Astronomía andalusí; Astronomía teórica; Cosmología medieval; Filosofía medieval; al-Andalus; Ŷābir b. Aflah; Ptolomeo; Almagesto; Ișlāh al-Maŷisțti. placed among the members of the Andalusian revolt against Ptolemaic astronomy. The final conclusion is that Jābir b. Aflah's criticisms are not themselves cosmological - although they may have cosmological implications - but of a completely technical mathematical nature. He should not, therefore, be placed among the members of the Andalusian revolt against Ptolemaic astronomy.

Key words: Astronomy; Greek Astronomy; Medieval Astronomy; Islamic Astronomy; Andalusian Astronomy; Theoretical Astronomy; Medieval Cosmology; Medieval Philosophy; al-Andalus; Jābir b. Aflah; Ptolemy; Almagest; Ișlāh al-Majisțti.

\section{Prolegomena}

\section{El astrónomo y matemático andalusí Ŷābir b. Aflah ${ }^{2}$ es conocido en la Historia de la Astronomía por su obra más importante, el Iṣlāh}

2 A continuación indicaremos la bibliografía básica para el estudio de la obra de Ŷābir b. Aflah. La introducción general más importante a Ŷābir b. Aflah es Lorch, R.P., "The Astronomy of Jābir b. Aflāh", Centaurus, 19 (1975), 85-107 (reimp. R.P. Lorch, Arabic Mathematical Sciences: Instruments, Text, Transmission, Aldershot, 1995, VI), producto de su tesis doctoral leída en la Universidad de Manchester en 1971: Jābir ibn Aflah and his Influence in the West. Este mismo autor ha tratado diferentes aspectos de la obra de Ŷābir b. Aflah en varios artículos, como el instrumento que se describe en el Ișlāh al-Maŷisțī, vid. Lorch, R.P., "The Astronomical Instruments of Jābir ibn Aflah and the Torquetum", Centaurus, 20 (1976), 11-34 (reimp. Arabic Mathematical Sciences, XVI), o la influencia de Ŷābir b. Aflah en la trigonometría europea, vid. "Jābir ibn Aflah and the Establishment of Trigonometry in the West", en Arabic Mathematical Sciences, VIII. Lorch ha estudiado asimismo la transmisión de los diversos manuscritos conservados en árabe, hebreo y latín, vid. "The Manuscripts of Jābir's Treatise", en Arabic Mathematical Sciences, VII. Otros investigadores han tratado aspectos adicionales de la obra de Ŷābir b. Aflah como el método que propone para demostrar la bisección de la excentricidad en los planetas superiores, estudiado por Swerdlow, N.M., "Jābir ibn Aflah's interesting method for finding the eccentricities and direction of the apsidal line of superior planets", en D.A. King y G. Saliba (eds.), From Deferent to Equant. A Volume of Studies in the History of Science in the Ancient and Medieval Near East in Honour of E.S. Kennedy, Nueva York, 1987, 501-512. H. Hugonnard-Roche ofrece las mismas conclusiones que Swerdlow en "La théorie astronomique selon Jābir ibn Aflah", en G. Swarup, A.K. Bag y K.S. Shukla (eds.), History of Oriental Astronomy. Proceedings of an [sic] International Astronomical Union Colloquium n. ${ }^{\circ} 91$ (1985), Cambridge, 1987, 207-208. Samsó ha estudiado las críticas de Ŷābir b. Aflah al modelo ptolemaico de Mercurio, vid. Samsó, J., "Ibn al-Haytham and Jābir b. Aflah's Criticism of Ptolemy's Determination of the Parameters of Mercury", Suhayl, 2 (2001), 199-225 (reimp. J. Samsó, Astronomy and

Al-Qanțara (AQ) XXX 1, enero-junio 2009, pp. 83-136 ISSN 0211-3589 
al-Mayisțī, en la que, entre otras críticas, refutó el orden de las esferas dado por Ptolomeo. Ŷābir b. Aflah situó las esferas de Mercurio y Venus por encima de la del Sol. Esta crítica tuvo un hondo impacto cosmológico. En paralelo, un grupo de filósofos andalusíes, más o menos contemporáneos de Ŷābir b. Aflah, elaboró una serie de críticas a la astronomía ptolemaica desde un punto de vista cosmológico. A.I. Sabra dio el nombre a este movimiento de «rebelión andalusí contra la astronomía ptolemaica».

En este trabajo me propongo responder a la pregunta de si cabe incluir la mencionada obra en la «rebelión andalusíł» descrita por Sabra.

Para ello, esbozaré primero en qué consistió la «rebelión andalusí» y, acto seguido, presentaré la figura de Ŷābir b. Aflah. A continuación, presentaré su obra principal, el Ișlāh al-Maŷisțì y, tras esta primera introducción, para determinar si Ŷābir b. Aflah perteneció a la rebelión anti-ptolemaica, analizaré en primer lugar la Introducción del Ișlāh al-Maŷisți, en la que el autor describe su intención y la génesis de la obra y, en segundo lugar, describiré el carácter de las diversas críticas a la astronomía ptolemaica que contiene el Ișlāh al-Maŷisțí. Por último, en apoyo a este estudio, presentaré una edición y traducción de la Introducción al Ișlāh al-Maŷisțī según los tres manuscritos árabes conservados.

Astrology in al-Andalus and the Maghrib, Aldershot-Burlington, 2007, VII). Otros trabajos dedicados al estudio de las críticas de Ŷābir b. Aflah son: Bellver, J., "Jābir b. Aflah on the four-eclipse method for finding the lunar period in anomaly", Suhayl, 6 (2006), 159-248; idem, "Ŷābir b. Aflah en torno a la inclinación de los eclipses en el horizonte", Archives Internationales d'Histoire des Sciences, 57, 158 (2007), 3-25; idem, "Jābir b. Aflah on lunar eclipses", Suhayl, 8 (2008), 47-91; idem, "Jābir b. Aflah on the lunar eccentricity and prosneusis at syzygies", Zeitschrift für Geschichte der Arabisch-Islamischen Wissenschaften, 18 (2008-2009), en prensa. Por último debemos señalar varios resúmenes de la obra de Ŷābir b. Aflah: Delambre resume las críticas de Ŷābir b. Aflah a Ptolomeo utilizando como base la traducción latina del Ișlāh al-Maŷisțì y lo considera un autor de escaso valor, vid. Delambre, M., Histoire de l'Astronomie du Moyen Age, París, 1819, 179-185 (reimp. Nueva York-Londres, 1965); Carmody edita la lista de errores en el Almagesto según la traducción latina del Ișlạh al-Maŷisți y da una imagen más favorable del autor, sobre todo por su influencia en al-Bitrūŷ̀i, vid. Carmody, F.J., al-Bitrūŷȳ, De Motibus Celorum, Berkeley-Los Ángeles, 1952, 29-32; y Samsó lo sitúa en el contexto de la astronomía andalusí post-zarqālí y en el inicio de las críticas a la astronomía teórica ptolemaica, vid. Samsó, J., Las ciencias de los antiguos en al-Andalus, Madrid, 1992, 317-320 y 326-330. El más reciente es Calvo, E., "Ibn Aflah", DAOA, Granada, 2002, I, 420-421.

Al-Qanțara (AQ) XXX 1, enero-junio 2009, pp. 83-136 ISSN 0211-3589 


\section{La rebelión andalusí contra la astronomía ptolemaica}

En 1984, A.I. Sabra, en su importante artículo «The Andalusian revolt against Ptolemaic astronomy» ${ }^{3}$, identificó una serie de autores andalusíes del s. XII que, a partir de objeciones de carácter filosófico, criticaron la astronomía matemática legada por Ptolomeo. Entre los autores que participan en esta corriente en al-Andalus cabe citar a Ibn Bāŷŷa (m. 537/1138), Ibn Țufayl (m. 581/1185), Ibn Rušd (m. 595/1198), Maimónides (m. 601/1204) y al-Bițūŷī (fl. 596/1200). Estos autores, a partir de una fuerte influencia del pensamiento filosófico de Aristóteles, consideran que una ciencia debe explicar las causas de los fenómenos y no sólo describirlos. La Astronomía, por tanto, debe dar cuenta de las causas de los fenómenos astronómicos y no sólo permitir la descripción matemática de los mismos. Así pues, los modelos astronómicos deben ajustarse a los postulados de la Física y, en especial, al tratamiento que la Física hace de los movimientos circulares. Pero puesto que, para estos autores, la Física la delineó Aristóteles ${ }^{4}$, una verdadera ciencia de los objetos celestes no puede contradecir la cosmología aristotélica ${ }^{5}$. Así pues, siguiendo al Estagirita, los objetos celestes únicamente pueden moverse describiendo un círculo ${ }^{6}$ con movimiento uniforme ${ }^{7}$. Además, las diversas esferas celestes no pueden tener su centro en algún otro punto que no sea el centro de la Tierra, es decir, deben ser homocéntricas ${ }^{8}$. La astronomía de Ptolomeo viola estas condiciones pues el ecuante viola el movimiento circular uniforme y la arquitectura de excéntricas y epiciclos viola el homocentrismo.

${ }^{3}$ Sabra, A.I., "The Andalusian Revolt against Ptolemaic Astronomy: Averroes and al-Bitrūjì”, en E. Mendelsohn (ed.), Transformation and Tradition in the Sciences: Essays in Honor of I. Bernard Cohen, Cambridge, 1984, 133-153.

${ }^{4}$ En torno a las características del movimiento circular en Aristóteles, cf. Aristóteles, Física, VIII. X.

${ }^{5}$ En torno a la cosmología de Aristóteles, la referencia básica es L. Elders, Aristotle's Cosmology. A commentary on the De Caelo, Assen, 1966.

${ }^{6}$ Cf. Aristóteles, De Caelo, I.II.

7 Cf. Aristóteles, De Caelo, II.VI.

8 En torno a la estructura homocéntrica de las esferas celestes, cf. Aristóteles, De Caelo, II.IX y Easterling, H.J., "Homocentric spheres in De Caelo", Phronesis, 6 (1961), 138-153.

Al-Qanțara (AQ) XXX 1, enero-junio 2009, pp. 83-136 ISSN 0211-3589 


\section{Ŷābir b. Aflạ̣ al-Išbīlī}

Abū Muḥammad Y̌ābir b. Aflaḥ al-Išbīlī, o al-Andalusī, conocido en el mundo latino como Geber filius Afflay Hispalensis ${ }^{9}$, fue un matemático y astrónomo teórico andalusí que floreció probablemente en Sevilla en la primera mitad del s. XII (primera mitad del s. VI de la Hégira). Apenas contamos con datos biográficos sobre su persona ${ }^{10}$, excepto una breve referencia de Maimónides (529-601/1135-1204) en su Guía de Perplejos, II, 9, donde el autor discute la posición de Venus y Mercurio en relación al Sol y menciona que conoció a su hijo ${ }^{11}$. Ibn Rušd (520-595/1126-98) menciona asimismo en su Mujtașar al-Maŷisțī que Ŷābir b. Aflah vivió en su mismo siglo, es decir, con posterioridad a 1106 (500h.). Además, de la cita anterior de Maimónides parece deducirse que Ibn Bāŷŷa (m. 537/1138) trató el orden de las esferas tras la publicación del libro del Ișlāh al-Maŷisțī, por lo que es muy plausible que Ŷābir b. Aflah estuviera activo al menos entre $500 / 1106$ y $537 / 1138^{12}$.

Si bien la fama de Ŷābir b. Aflah descansa básicamente sobre la obra que se ha convenido en llamar Ișlāh al-Maŷisțtī ${ }^{13}$, el autor elaboró algunos tratados menores de carácter matemático. Su opera minora consiste en:

I. El tratado conservado en hebreo Ha-dibbur be temunah ha-hittukit (Comentario de la Figura Sectoris de Menelao) traducido

9 Ŷābir b. Aflah ha sido confundido con otros autores como el otro Geber latino, es decir el alquimista Ŷābir b. Hayyān (prob. s. II/VIII), el astrónomo Muhammad b. Ȳābir al-Battānī (ca. 858-929), el poeta bagdadí Abū l-Qāsim 'Alī b. Aflah al-Afsī (m. 535/1141), el astrónomo judío Abū Aflah ha-Saraqosțī (fl. s. VII/XIII o VIII/XIV), o el traductor conocido como Juan de Sevilla o Johannes Hispalensis.

10 Ȳābir b. Aflah al-Išbīlī no aparece en ningún repertorio bio-bibliográfico estudiado por Meouak en su intento de reconstrucción de un hipotético linaje de los Banū Aflah andalusíes. Cf. Meouak, M., "Los Banū Aflạ̣: una hipotética familia de funcionarios y letrados andaluces", en M.L. Ávila (ed.), EOBA, Granada, 1989, II, 101-117.

${ }_{11}$ Maimonides, M., The Guide of the Perplexed, S. Pines (trad.), Chicago-Londres, 1963, 268-9. Hay traducción española por D. Gonzalo Maeso, Guia de Perplejos, Madrid, 2005, 258-259. En esta cita, Maimónides sitúa a Ŷābir b. Aflạ entre un grupo de matemáticos críticos con el orden de las esferas que da Ptolomeo.

12 Para la datación de Ŷābir b. Aflah, cf. Lorch, "The Astronomy of Jābir b. Aflāḥ”, 85-86.

13 Seguimos a Lorch, "The Astronomy of Jābir b. Aflāḥ", 88 en su adopción de este título para esta obra a partir del Ms. Berlín Ar. 5653.

Al-Qanțara (AQ) XXX 1, enero-junio 2009, pp. 83-136 ISSN 0211-3589 
por Qalonymos ben Qalonymos. Se trata de un pequeño comentario al tratado de Tāabit b. Qurra (836-931) sobre el teorema de Menelao ${ }^{14}$.

II. El tratado conservado en hebreo Comentario a las Esféricas de Menelao que, en dos manuscritos, se halla a continuación del Comentario de la Figura Sectoris de Menelao que acabamos de mencionar ${ }^{15}$.

III. Un breve fragmento de un comentario a Euclides mencionado por Kapp ${ }^{16}$.

IV. El manuscrito latino con incipit «Omnium recte philosophantium uerisimilibus coniecturis credibilibus argumentis, sed et firmissimis rationibus comprehensio est forman celi sphericam esse, motumque ipsius orbicularem...» descrito por Millás Vallicrosa ${ }^{17}$, que Thorndike y Kibre atribuyen, siguiendo la adscripción de diversos manuscritos, a Ȳābir b. Aflaḥ ${ }^{18}$.

\section{El Iṣlāh al-Maŷisṭ̂̄}

El Ișlāh al-Maŷisțī (Corrección del Almagesto) es una obra en nueve libros, única en su tipo en la astronomía andalusí en la medida en que propone reescribir y mejorar el Almagesto. En ella, Ȳābir b. Aflah se propone básicamente hacer accesible a sus contemporáneos el Almagesto. Para ello, lo reescribe, simplificando su matemática, y lo dota de una introducción a la trigonometría ${ }^{19}$. Esta introducción tiene gran importancia histórica, pues sirvió, gracias a su traducción al latín, de vía de entrada de la trigonometría esférica basada en la Re-

${ }^{14}$ Para un resumen del comentario de Ŷābir b. Aflah, cf. Lorch, R.P., Thābit ibn Qurra, On the Sector-Figure and Related Texts. Edited with Translation and Commentary, Frankfurt am Main, 2001, 387-390.

15 Mss. Bodleian Hunt. 96, fols. 42r y ss. y Heb. d 4, fols. 169r-177r.

16 Ms. Berlin Q 747, fols. 133v-134v referido en Kapp, A.G., "Arabische Übersetzer und Kommentatoren Euklids, sowie deren math.-naturwiss. Werke auf Grund des Ta 'rīkh al-Hukamā' des Ibn al-Qifți: II", Isis, 23, 1 (1935), 71. Cf. Lorch, "The Astronomy of Jäbir b. Aflāh", 106 n. 55.

17 Millás Vallicrosa, J.M., Las traducciones orientales en los manuscritos de la Biblioteca Catedral de Toledo, Barcelona, 1942, 203-204. Este Ms. corresponde al XLII de su relación, fols. $67 \mathrm{r}-80 \mathrm{v}$.

18 Thorndike, L. y Kibre, P., Catalogue of Incipits of Mediaeval Scientific Writings in Latin, Cambridge (MA), 1937, col. 470.

${ }_{19}$ Para una lista de los diversos comentarios árabes del Almagesto o de aspectos particulares del mismo, cf. F. Sezgin, Geschichte des Arabischen Schrifttums, Leiden, 1978, VI, 90-94.

Al-Qanțara (AQ) XXX 1, enero-junio 2009, pp. 83-136 ISSN 0211-3589 
gla de las cuatro cantidades en el Occidente latino ${ }^{20}$. Además, Ŷābir b. Aflah, en su reedición del Almagesto, introduce algunas críticas a la obra de Ptolomeo. La existencia de las críticas al Almagesto y la proximidad geográfica y temporal del autor respecto a los autores de la revolución anti-ptolemaica andalusí del s. XII, han llevado a considerarlo como un posible precedente de estos autores. El papel del Ișlāh al-Maŷisțī en la introducción en el Occidente latino de la trigonometría y las críticas que contiene a la astronomía de Ptolomeo sitúan a esta obra en un lugar importante en la historiografía de la ciencia contemporánea. Sin embargo, estos dos aspectos confieren un cierto sesgo a nuestra comprensión de la obra respecto a la intención que el autor tuvo al escribirla.

Conservamos únicamente tres manuscritos árabes en alfabeto árabe y tres aljamiados hebraico-árabes ${ }^{21}$, lo que, junto al reducido número de citas en la bibliografía árabe, muestra que la difusión del Ișlāh al-Maŷisțī en el mundo árabe fue más bien escasa ${ }^{22}$. Los tres manuscritos árabes transmiten dos redacciones diferentes: (i.) la redacción del Ms. Escorial 910, al que aludiremos a partir de ahora como Es ${ }^{1}$; y (ii.) la redacción del Ms. Berlín 5653, al que aludiremos como B. El tercer manuscrito, el Ms. Escorial 930, al que aludiremos como Es², mezcla contenidos de las dos redacciones anteriores. Lorch considera, a partir de un estudio de la introducción trigonometrica del Ișlāh al-Maŷistị, que la redacción del Ms. Es ${ }^{1}$ es más sucinta y más completa ${ }^{23}$, lo que concuerda con una versión corregida. Sin embargo, el Ms. Berlín 5653 es más correcto desde un punto de vista astronómico ${ }^{24}$.

Ibn al-Qifțī (568-646/1172-1248), en su Ta'rïj al-hukamä', menciona que Joseph ben Jehuda (m. 623/1226), discípulo de Maimónides, llevó consigo la Astronomía de Ŷābir b. Aflaḥ (el Ișlāh al-Maŷisțî) de Ceuta a Fustāṭ, donde permaneció un período no muy largo antes de marchar a Alepo en 583/1187, lugar en el que fijó su residencia defini-

20 Lorch, "Jābir ibn Aflah".

${ }^{21}$ Lorch, "The Astronomy of Jābir b. Aflāḥ”, 88 y “The Manuscripts of Jābir's Treatise", 1.

22 Hāŷŷŷ Jalīfa menciona el Ișlāḥ al-Maŷisțī en su Kašf al-zunūn dando únicamente el incipit, pero no el nombre de la obra ni del autor. Cf. Jalīfa, Ḥ, Kašf al-ẓnūn 'an asāmī al-kutub wa-l-funūn, Teherán, 1967, 2, col. 1595.

${ }^{23}$ Lorch, "The Astronomy of Jābir b. Aflāh", 88.

${ }^{24}$ Por ejemplo, en el cálculo de la longitud de la conjunción aparente a partir de la verdadera en los eclipses de Sol. Cf. B. fol. $67 \mathrm{v}$ respecto a $\mathrm{Es}^{1}$ fol. $65 \mathrm{v}$. 
tiva. Durante su estancia en Fustạt junto a Maimónides, éste le pidió que la corrigiera ${ }^{25}$. Se ha sugerido que las dos redacciones correspondan a la versión original de Ŷābir b. Aflah y a la corrección de Joseph ben Jehuda y Maimónides. Lorch considera probable que la redacción del Ms. Escorial 910 corresponda a la corrección llevada a cabo por Joseph ben Jehuda y Maimónides ${ }^{26}$. Sin embargo, posteriormente, siguiendo sugerencias de Saliba ${ }^{27}$, apunta la posibilidad de que ambas redacciones fueran sucesivas versiones del Ișläh al-Maŷisțī llevadas a cabo por el propio Ŷābir b. Aflah ${ }^{28}$, si bien Lorch no descarta la primera posibilidad. Si esto fuera así, los errores que acarrea esta redacción deberían de tener su origen en algún copista posterior. En 2007, Saliba sugirió que la redacción del Ms. Berlín 5653 corresponda de hecho a una segunda redacción de la del Ms. Escorial $910{ }^{29}$. Esta tesis explicaría las mejoras en el contenido astronómico de la redacción del Ms. de Berlín. En cualquier caso, parece plausible que el Ms. Berlín 5653, copiado en Damasco en 626/1229, proceda en última instancia del manuscrito llevado por Joseph ben Jehuda (m. en Alepo en 623/1226) en su salida de Ceuta, pues al coincidir con la traducción de Gerardo de Cremona (m. 583/1187) el Ms. Berlín 5653 no puede corresponder a la versión de Joseph ben Jehuda, llevada a cabo durante su corta estancia en Fusțāt que concluyó en 583/1187.

Si la difusión del Ișlāh al-Maŷisțī en la astronomía árabe fue escasa, en la astronomía en lengua latina y hebrea fue mucho mayor. Gerardo de Cremona (ca. 507-583/ca. 1114-1187) lo tradujo al latín. Lorch señala que es muy probable que la traducción del Ișlāh al-Maŷisțī al latín se llevara a cabo antes que la del Almagesto ${ }^{30}$, pues las citas del Almagesto que aparecen en la traducción latina del Ișlāh al-Maŷisțī no coinciden con la traducción del Almagesto de Gerardo de Cremona ${ }^{31}$. Pues-

25 Ibn al-Qifțī, Ta'rīj al-hukamā', Lippert (ed.), Leipzig, 1903, 392-393. La misma noticia con una redacción muy aproximada se encuentra en Ibn al-'Ibrī (Barhebraeus), Tã'rīj mujtaṣar al-duwal, Dār al-kitāb al-'ilmiyya, Beirut, 1997, 150.

26 Lorch, "The Astronomy of Jābir b. Aflāḥ", 89.

${ }^{27}$ Cf. Lorch, "Jābir ibn Aflah", 34. Saliba sugirió esta posibilidad en el 5th International Symposium for the History of Arabic Science.

${ }^{28}$ Lorch, "Jābir ibn Aflah", 34.

29 Saliba sugirió esta idea en una intervención en el congreso de Barcelona de 2007: A Shared Legacy: Islamic Science East and West-Barcelona Conference.

${ }^{30}$ Lorch, "The Astronomy of Jābir b. Aflāh", 91.

31 Se conservan 27 Mss. latinos, dos de ellos en Cracovia y otros dos copiados por Regiomontanus. Existe impresión de la traducción de Gerardo de Cremona en Petrus 
to que la traducción del Almagesto se llevó a cabo antes de 570/1175 32, tiene sentido pensar que la del Ișlāh al-Maŷisțī sea anterior a esta fecha. La traducción de Gerardo de Cremona sigue fielmente la redacción del Ms. Berlín 5653, si bien difiere en algunos detalles. En primer lugar, el instrumento con el que Ŷābir b. Aflah sustituye los instrumentos que aparecen en el Almagesto es ligeramente diferente entre las versiones latina y árabe. En la versión árabe, este instrumento es más próximo al torquetum $\mathrm{y}$, en la latina, el método para la medida de latitudes es más próximo al triquetrum ${ }^{33}$. Puesto que el instrumento que se describe en la introducción del Iṣlăḥ al-Maŷisțī es el que aparece en las dos redacciones en árabe conservadas - es decir, el más próximo al torquetum - , pero por otra parte el instrumento más próximo al triquetrum aparece en la revisión en hebreo de Samuel ben Jehuda de Marsella ( $f l$. 735/1335) (con lo que es también de origen árabe), el manuscrito árabe sobre el que Gerardo de Cremona trabajó para traducir el Ișlāh al-Maŷisți al latín era ya una versión revisada, quizá únicamente en este punto, de la obra original de Ŷābir b. Aflah. Otra diferencia importante se encuentra en el libro VI de la traducción latina del Ișlāh al-Maŷisțī, pues contiene un fragmento dedicado a la Vía Láctea que no aparece en las redacciones en árabe. Por último, obviamente el prefacio de carácter religioso que aparece en la versión árabe se ha omitido en la traducción latina.

Tras la traducción latina, se llevaron a cabo dos traducciones adicionales al hebreo. En primer lugar, en 672/1274 la tradujo Moše ibn Tibbon $\left(f l\right.$. entre $637 / 1240$ y 682/1283) ${ }^{34}$. Esta traducción incluye el ins-

Apianus, Instrumentum primi mobilis. Accedunt iis Gebrii filii Affla Hispalensis Astronomi vetustissimi pariter et peritissimi, libri IX de astronomia, ante aliquot secula Arabice scripti, et per Giriardum Cremonensem latinitate donati, nunc vero omnium primum in lucem editi, Nuremberg, 1534.

${ }^{32}$ Un húngaro llamado Thaddeus copió un manuscrito de la traducción del Almagesto de Gerardo de Cremona en 1175. Cf. MS Florenciae, Biblioteca Medicea Laurenziana, 89, sup. 45; véase Kunitsch, P., Ptolemy. Der Sternkatalog des Almagest, Wiesbaden, 1986-1991, I, 16.

${ }^{33}$ La referencia fundamental para el conocimiento de este instrumento es Lorch, "The Astronomical Instruments", donde edita los textos árabe y latino. Para la diferencia entre los instrumentos en la versión árabe y latina, cf. ibidem, 30. Véase también Samsó, Las ciencias de los antiguos, 317-320.

${ }^{34}$ En torno a Moše ibn Tibbon, cf. Steinschneider, M., Jewish Literature from the Eight to the Eighteenth Century with an Introduction on Talmud and Midrash. An Historical Essay, Londres, 1857, 96, 104, 125, 167, 184, 197; Renan, E. y Neubauer, A., "Les Rabbins Française", en Histoire littéraire de la France, XXVII, París, 1877 (reimp. Re- 
trumento que aparece en la traducción latina. El único manuscrito conservado de esta traducción ${ }^{35}$ es probablemente un autógrafo pues en el colofón encontramos "And I have translated it from Arabic language into Hebrew language, I, Moses ben Rabbi Samuel ibn Tibbon the Spaniard» ${ }^{36}$.

Jacob ben Mahir ibn Tibbon (633-703/1236-1304) ${ }^{37}$, conocido también por los latinos como Profatius Judaeus, llevó a cabo una segunda traducción al hebreo del Ișlāh al-Maŷisțī. Samuel ben Jehuda de Marsella (fl. 735/1335) ${ }^{38}$ revisó la traducción de Jacob ben Mahir ibn Tibbon. Él y su hermano David intentaron llevar a cabo una traducción de un manuscrito árabe que encontró en Trinquetailles, un suburbio de Arlés ${ }^{39}$. Pudieron copiar una octava parte del texto durante dos días, pero tuvieron que devolver el manuscrito y con las prisas se les desordenaron las páginas copiadas. Tras cierto tiempo, Samuel ben Jehuda de Marsella pudo encontrar una versión de la

nan, E., Les Rabbins Français du commencement du quatorzième siècle, 1, Farnborough, 1969) 593 y ss., 750 y ss.; Ibidem, "Les Ecrivains Juifs Français", en Historie littéraire de la France, XXXI, París, 1893 (reimp. Renan, E., Les Rabbins Français du commencement du quatorzième siècle, 2, Farnborough, 1970) 356, 432, 686, 759; Graetz, H., Geschichte der Juden von den Aufängen bis auf die Gegenwart, 1853-1875, VII, Leipzig, 1863, 103; Winter, J. y Wünsche, A., Die Jüdische Litteratur seit Abschluss de Kanous. Eine Prosaische und poetische Authologie mit biographischen und literatur geschichlichen Einleitungeu, Trier, 1894-1896, III, 661; Gross, H., Gallia Judaica, París, 1897, 59, $327,356,373$, 534; y por último Sarton, G., Introduction to the History of Science, Baltimore, 1927-1948 (reimp. Malabar, 1975), II, 847-850 da la lista de sus traducciones.

35 Bodleian, Opp. Add. Fol. 17, fols. 1-100r.

36 Traducción de Lorch; cf. Lorch, "The Astronomy of Jābir b. Aflāḥ", 93.

37 En torno a Jacob ben Mahir ibn Tibbon, cf. Munk, Mélanges de philosophie Juive et Arabe, París, 1859, 489; Carmoly, Histoire des Médecins Juifs, Anciens et Modernes, Bruselas, 1844, 90; Steinschneider, Catalogus Librorum Hebracorum in Bibliotheca Bodleiana, Berlín, 1852-60, col. 1232; idem, Die Hebräischen Übersetzungen des Mittelalters und die Juden als Dolwetscher: Ein Beitrag zur Literaturgeschichte des Mittelalters; meistenteils nach Handschriftlichen Quellen, Berlín, 1893, 54, 56, 144, 503-511, 516, 544, 607-608, 813; Graetz, Geschichte der Juden, VII, 246; Renan-Neubauer, "Les Rabbins Français", 599 y ss.; Gross, Gallia Judaica, 332.I. Br.; Sarton, Introduction to the History of Science, II, 850-853.

38 En torno a Samuel ben Jehuda de Marsella, cf. Steinschneider, Die Hebräischen Übersetzungen, 106, 122, 152, 544; Renan-Neubauer, "Les Ecrivains Juifs Français", 207; Gross, Gallia Judaica, 379; Sarton, Introduction to the History of Science, III, 128. En torno a la labor traductora de Samuel ben Judah de Marsella, cf. Berman, L.V., "Greek into Hebrew: Samuel ben Judah of Marseilles, Fourteenth-Century Philosopher and Translator", en A. Altmann (ed.), Jewish Medieval and Renaissance Studies, Cambridge (MA), 1967, 289-320.

39 Para esta noticia, cf. Lorch, "The Astronomy of Jābir b. Aflāḥ", 93.

Al-Qanțara (AQ) XXX 1, enero-junio 2009, pp. 83-136 ISSN 0211-3589 
traducción de Jacob ben Mahir ibn Tibbon, si bien la encontró defectuosa. Samuel ben Jehuda conocía sólo de oídas la traducción llevada a cabo por Moše ibn Tibbon, y corrigió la traducción de Jacob ben Mahir ibn Tibbon, pues encontró que faltaba el pasaje en torno a la Vía Láctea y la Esfera, siguiendo el manuscrito árabe que tiempo atrás intentaron copiar él y su hermano. Terminó la revisión en Aix en Provence en 735/1335 ${ }^{40}$. La revisión de Samuel ben Jehuda sigue en algunos momentos la redacción del Ms. Es ${ }^{1}$. En esta traducción aparecen los dos instrumentos. Toda esta serie de diferencias lleva a sugerir la hipótesis de que Jacob ben Mahir ibn Tibbon elaborara su traducción a partir de un manuscrito árabe similar al Ms. Es ${ }^{1}$, mientras que Samuel ben Jehuda de Marsella debió de cotejar la traducción de Jacob ben Mahir con un manuscrito similar al utilizado por Gerardo de Cremona en su traducción al latín. Esta hipótesis explicaría que la revisión de Samuel ben Jehuda contenga los dos instrumentos, así como los fragmentos que no aparecen en la redacción del Ms. Es ${ }^{1}$. También explicaría que la revisión de Samuel ben Jehuda siga en algunas ocasiones el orden de la versión revisada de la redacción del Ms. Es ${ }^{1}$.

\section{La Introducción del Iṣlāḥ al-Maŷisṭ̂̄}

En la Introducción del Iṣlāh al-Maŷisțī, Ŷābir b. Aflaḥ trata a grandes rasgos tres temas: (i.) la importancia de la astronomía matemática y su lugar en la jerarquía de las ciencias; (ii.) el lugar del Almagesto en el marco de la Astronomía, así como las dificultades que entraña para sus contemporáneos; $y$ (iii.) la soluciones que propone que le llevan a escribir el Ișlāh al-Maŷisțī.

\section{Concepto de la Astronomía y lugar que ocupa entre las ciencias}

En primer lugar, en el proemio en alabanza a Dios y bendición al Profeta, Ŷābir b. Aflah justifica el valor del intelecto y la ciencia mediante una serie de citas y referencias coránicas tendentes a establecer una correlación entre el papel del Profeta y el del intelecto $(\S 1 \text { y } § 2)^{41}$. 1036

${ }^{40}$ La traducción revisada se encuentra en los Mss. París BN. n. ${ }^{\text {os }} 1014,1024,1025$,

${ }^{41}$ Las llamadas hacen referencia a los párrafos de la traducción y edición de la Introducción del Ișlāḥ al-Maŷisțī que se incluye al final del presente artículo.

Al-Qantara (AQ) XXX 1, enero-junio 2009, pp. 83-136 ISSN 0211-3589 
A continuación, divide la ciencia en dos grandes áreas: la ciencia de la Ley revelada ('ilm al-šarī'a) que ocupa el nivel más noble entre las ciencias, y el resto (§3). Esta clasificación deriva probablemente de la correlación que acaba de establecer entre el Profeta y el intelecto. Del Profeta, se derivaría la ciencia de la Ley revelada, y en general cualquier ciencia de corte naqli. Del intelecto derivarían las diversas ciencias de corte 'aqli. Llegado a este punto, y tras garantizar la precedencia de la ciencia de la Ley revelada sobre cualquier otra ciencia, se centra en la descripción de las características que garantizan la nobleza de una ciencia intelectual (§3). La ciencia más noble es aquella que cumple que (i.) sus contenidos sean estables e imperecederos, y (ii.) su método garantice la certeza (țruq yaqiniyya) y aleje las dudas. A continuación, examina si la Astronomía ('ilm al-hay'a) coincide con esta descripción. Para ello, define en primer lugar el contenido de la Astronomía (§4). Dice Ŷābir b. Aflah:

La ciencia de la Astronomía ( 'ilm al-hay'a, lit. "ciencia de la estructura") consiste en [el estudio de] los movimientos del Sol, de la Luna, de las estrellas $(\text { nuŷūm })^{42}$ y en el conocimiento de sus esferas (aflāk) (§4).

A continuación, señala que la ciencia de la Astronomía cumple efectivamente los dos criterios anteriores pues, (i.) los contenidos «son estables e imperecederos sin que sufran ningún cambio, mientras Dios, sea ensalzado, se lo conceda» ${ }^{43}$ y ello lo deduce de la definición que ha dado de Astronomía al tratarse los movimientos celestes de movimientos armónicos, y (ii.) los métodos (țuruq) garantizan la certeza y son claros (turuq yaqiniyya bayyina). Ȳābir b. Aflạ̣ toma de Ptolomeo estas ideas si bien no cita literalmente el Almagesto ${ }^{44}$. Ptolomeo, siguiendo a Aristóteles, divide la filosofía teorética en tres partes: Teología, Matemáticas y Física. La Teología — dice Ptolomeo- estudia el motor inmóvil del universo, separado por completo de la realidad visible. La Física estudia la naturaleza material y móvil. Las Matemáticas, en cambio, estudian la naturaleza en cuanto a la forma, número, tamaño, lugar, tiempo, etc.; es decir, magnitudes cuantificables. Ptolomeo considera

42 Ptolomeo incluye a veces en el término "estrellas" los planetas y en algunas ocasiones el Sol y la Luna. Cf. la traducción inglesa de Toomer, G.J., Ptolemy's Almagest, London, 1984 (en adelante, PtA), 37 n. 8.

43 Ibidem.

${ }^{44}$ Cf. Almagesto 1.1 (PtA, 35-36).

Al-Qanțara (AQ) XXX 1, enero-junio 2009, pp. 83-136 ISSN 0211-3589 
que únicamente se puede obtener un conocimiento objetivo de las Matemáticas pues la Teología se ocupa de un ámbito que, por naturaleza, es invisible e intangible, mientras que la Física se ocupa de la materia que, por naturaleza, es opaca e inestable. En cambio, el método de las Matemáticas, ciencia dentro de la que cabe situar a la Astronomía, es indiscutible. Según Ptolomeo:

Only mathematics can provide sure and unshakeable knowledge to its devotees, provided one approaches it rigorously. For its kind of proof proceeds by indisputable methods, namely arithmetic and geometry. ${ }^{45}$

Y continúa señalando que, al poder apoyarse en un método incuestionable, orientó su investigación a aquellos objetos de estudio cuya naturaleza fuera eterna y que no estuvieran sujetos a cambio. Dice Ptolomeo:

Hence we were drawn to the investigation of that part of theoretical philosophy, as far as we were able to the whole of it, but especially to the theory concerning divine and heavenly things. For that alone is devoted to the investigation of the eternally unchanging. For that reason it too can be eternal and unchanging (which is a proper attribute of knowledge) in its own domain, which is neither unclear nor disorderly. ${ }^{46}$

En las dos citas anteriores encontramos las dos características con las que Ŷābir b. Aflaḥ ha definido la ciencia más noble al margen de las ciencias religiosas: (i.) contenidos estables e invariables y (ii.) método indudable; si bien estas citas no se encuentran recogidas literalmente en el Iṣlāh al-Maŷisțī.

\section{Valoración de Ptolomeo y análisis de las dificultades ante el estudio del Almagesto}

Tras tratar la definición y lugar de la Astronomía, Ŷābir b. Aflah se centra en la figura que acrisoló la tradición astronómica de los antiguos (al-qudamā' qabla-hu) y que enriqueció con sus propios conocimientos: Ptolomeo ( 55$)$. Su juicio en torno al Almagesto en esta introducción sigue tres ideas básicas:

- tiene una opinión sobre la obra en general muy positiva ${ }^{47}$;

45 Ibidem.

46 Ibidem.

47 Este tratamiento muy positivo de Ptolomeo en la Introducción contrasta, por el Al-Qanțara (AQ) XXX 1, enero-junio 2009, pp. 83-136 ISSN 0211-3589 
- llama la atención sobre su dificultad para el estudiante;

- e indica una serie de errores que comete.

$\hat{Y}$ ābir b. Aflah señala que el Almagesto es suficiente para el conocimiento cabal de la Astronomía pues reúne todos los temas. Además, como ya hemos mencionado, sintetiza la tradición astronómica de los antiguos y la hace progresar llevándola a su perfección (qad kumila $h \bar{a} \underline{d} \bar{a} l$ - 'ilm 'inda- $h u)(\S 5)^{48}$. Todo ello supone «una bendición generosa y de grandes proporciones» (§5). El Almagesto es un libro inmenso (kitāb 'azìm) (§6).

Ahora bien, su opinión se empaña cuando considera el Almagesto desde el punto de vista pedagógico. Ptolomeo señala en su prefacio al Almagesto que ha adaptado la forma de exposición de sus descubrimientos a los que ya hayan progresado en este campo. Es decir, se dirige a un lector con cierto grado de competencia ${ }^{49}$. Y̌ābir b. Aflah, en cambio, piensa en un lector del Almagesto sólo familiarizado con los Elementos de Euclides y desconocedor de la Astronomía, que se adentra por primera vez en el estudio de esta ciencia de la mano del Almagesto. Para este lector del Almagesto, Ŷābir b. Aflah señala una serie de dificultades que dividiremos en intrínsecas y extrínsecas a la propia obra de Ptolomeo. Dificultades intrínsecas:

- Ptolomeo mezcla en el Almagesto contenidos de corte teórico (métodos y demostraciones) con contenidos de corte práctico (cálculos y elaboración de tablas). Este hecho provoca que los contenidos teóricos queden dispersos a lo largo de la obra dificultando su estudio. Ŷābir b. Aflah señala la existencia de diversos motivos que hacen inútiles los contenidos prácticos del Almagesto pero no explicita cuáles son. Además, de modo extrínseco al propio Almagesto, la literatura de azyāŷy cubre la necesidad de una Astronomía de corte práctico y hace por tanto innecesario su tratamiento en el Almagesto ( $\$ 5.1)$.

- Algunos de los procedimientos y demostraciones se hallan muy resumidos en el Almagesto, lo que dificulta su comprensión (\$5.4).

Dificultades extrínsecas, no achacables a la propia obra, que podemos resumir en dos puntos:

contrario, con un tratamiento muy agrio a lo largo del resto de la obra; cf., por ejemplo, Bellver, "Jābir b. Aflah on the four-eclipse method", 190-191.

48 Esta referencia sólo se encuentra en el Ms. Es ${ }^{2}$.

49 Cf. Almagesto I.1 (PtA, 37) y la introducción de Toomer en PtA, 6. Al-Qanțara (AQ) XXX 1, enero-junio 2009, pp. 83-136 ISSN 0211-3589 
- El avance de la trigonometría esférica en la época de Ȳābir b. Aflah respecto a la época de Ptolomeo permite simplificar la matemática subyacente al Almagesto evitando el uso de razones compuestas de seis elementos como el teorema de Menelao (al-Šakl al-qatțā). Así pues, la nueva trigonometría esférica hace innecesario que el alumno se detenga en el estudio del Libro de Menelao y del Libro de Teodosio como paso previo al estudio del Almagesto ( $\$ 5.2$ y 5.3).

- La traducción del Almagesto al árabe ha podido introducir problemas textuales adicionales dificultando aún más el texto. De hecho, como veremos a lo largo del presente estudio, las dificultades que $\hat{Y}$ ābir b. Aflạ achaca a la traducción pueden deberse en mayor medida a defectos en el manuscrito del Almagesto que manejara (§5.5).

Estos problemas dificultan el acceso del estudiante, lo que supone que el tiempo dedicado se prolongue innecesariamente. Por este motivo, el estudiante puede caer en el desinterés, la desgana y la pereza.

\section{Ŷābir b. Aflaḥ y sus intenciones en la redacción del Ișlāḥ al-Maŷisțī}

Ŷābir b. Aflah explica en su introducción la motivación de su acercamiento al Almagesto, su proceso de estudio y la intención que le llevó a redactar el Ișlāh al-Maŷisțī:

Nos aconteció que dado el amor y la pasión por esta ciencia debido a las cosas que mencionamos y dado que, también, [Ptolomeo] había mencionado en el comienzo de su libro las razones que llevan a la pasión y el amor por ella no pudimos dejar de estudiarlo con atención y sobrellevamos las penalidades y dificultades que sufre su lector hasta que, gracias a Dios, sea bendito y ensalzado, comprendimos todo el contenido de este libro en torno a la astronomía ('ilm al-hay'a). Después de esto, no dejamos de persistir en el estudio y continuamos la investigación y la reflexión para que pudiera facilitarse [al estudiante satisfacer su] anhelo de esta ciencia enorme y acercar la comprensión de este libro inmenso (\$6).

El fragmento anterior contiene elementos importantes que permiten comprender el Ișlāh al-Maŷisțì y que se hace necesario subrayar:

- En primer lugar, Ŷābir b. Aflah identifica, al menos en un estadio inicial de estudio, Almagesto y Astronomía.

- Ŷābir b. Aflah parece estudiar solo el Almagesto y sólo el Almagesto. Por lo tanto, Ŷābir b. Aflah no parece tener amplia formación astronómica previa. El estudio de su obra muestra claramente, en cambio, que sí tenía formación matemática. 
— Ŷābir b. Aflah comprende todo el Almagesto, pese a las dificultades.

— Ŷābir b. Aflah, guiado por una motivación pedagógica, continúa su estudio e investiga sobre cómo facilitar a nuevos estudiantes el acceso al Almagesto, lo que le lleva a reescribir la obra.

Es importante subrayar que el interés de Ŷābir b. Aflah se centra en el Almagesto, de tal forma que la Astronomía queda en un segundo plano confundida con la obra de Ptolomeo. Así pues, Ŷābir b. Aflaḥ no pretende escribir una nueva summa astronómica de su tiempo equivalente a lo que fue en su día el Almagesto, con lo que necesariamente debería haber incorporado los nuevos desarrollos posptolemaicos. El autor únicamente desea escribir una segunda edición del Almagesto para hacerlo inteligible a un público contemporáneo con conocimientos básicos de matemáticas. Los contenidos astronómicos en este Almagesto (2. ${ }^{\text {a }}$ ed. corregida y depurada por Ŷābir b. Aflah, Sevilla, ca. 1100) son los mismos que aparecen en la primera edición ya que se edita desde un punto de vista básicamente matemático y con intención pedagógica.

A partir del cotejo del texto, así como de las indicaciones que aparecen en su Introducción, podemos considerar que son tres los criterios de edición que aplica:

I. En primer lugar, desea simplificar el texto;

II. desea asimismo subsanar las deficiencias motivadas por aproximaciones matemáticas o por una redacción en exceso sintética; y

III. desea corregir las incoherencias que se hallen en el textus receptus respecto a las premisas establecidas por el propio Ptolomeo.

A continuación, estudiaremos cómo aplica estos criterios y acto seguido nos centraremos en el estudio de algunos aspectos en los que ha sobresalido el Ișlāh al-Maŷisțī, como su tratamiento de la trigonometría, etc.

Con el objeto de simplificar el texto - criterio inicial de edición de nuestra relación_-, en primer lugar, Ŷābir b. Aflah elabora un primer libro centrado únicamente en el estudio de la trigonometría plana y esférica $(\$ 7, \S 8$ y $\S 12)$. En lo que respecta a la trigonometría plana, presenta esencialmente la trigonometría del Almagesto basada en la función cuerda. Pero, en cambio, su trigonometría esférica descansa principalmente en el teorema del seno ${ }^{50}$. En cualquier caso, este pri-

50 Cf. Lorch, "Jābir ibn Aflạ̣”, para un estudio de su trigonometría y su influencia en Occidente.

Al-Qanțara (AQ) XXX 1, enero-junio 2009, pp. 83-136 ISSN 0211-3589 
mer libro pretende agrupar todas las demostraciones geométricas necesarias a lo largo del Almagesto. Con ello, su intención es simplificar las demostraciones específicamente astronómicas al liberarlas durante su desarrollo de sus justificaciones geométricas.

En segundo lugar, el autor del Iṣlāḥ al-Maŷisțī, en su intención simplificadora, se propone eliminar la vertiente práctica del Almagesto $(\S 12)$. Únicamente trabaja desde un punto de vista geométrico con los diversos puntos significativos de la demostración, pero sin atender a los valores que intervienen en los cálculos. Quizá al final dé un valor numérico o señale el valor de un error cometido por Ptolomeo. Este último caso muestra que en ocasiones, pese a no consignar los cálculos en la demostración, sí que los ha efectuado por su parte ${ }^{51}$. En este sentido, debe señalarse que el Ișlāḥ al-Maŷisțī es un libro de astronomía que carece por completo de tablas. Ello no quiere decir que los procedimientos para el cálculo de las mismas no aparezcan, pues, en ocasiones, estudia la función que podría dar lugar a una tabla en el caso de que se calculase el valor resultante para cada argumento ${ }^{52}$.

Un caso especial dentro de este espíritu simplificador que anima su reedición es la elaboración de un instrumento con el que sintetiza los que aparecen en el Almagesto ${ }^{53}$. En su introducción señala:

[Ptolomeo] utilizó para las observaciones que necesitaba cuatro instrumentos con ocho armillas. Se nos ocurrió un único instrumento que no necesita sino una armilla, un cuadrante de otra y una regla que permite prescindir de todos los instrumentos que menciona ( $(9)$.

Este instrumento, que quizá no se construyó nunca, ha sido puesto en relación con el torquetum o torquet. Debe señalarse que la aparición de este instrumento en el Ișlāh al-Maŷisți no supone una contradicción del espíritu teórico que anima su redacción ${ }^{54}$. La simplificación de la vertiente práctica que acabamos de señalar se refiere únicamente a su aproximación metodológica. Pero en este caso, Ŷābir b. Aflah considera que este instrumento forma parte de los contenidos y no de los métodos de exposición. Puesto que Ŷābir b. Aflah preten-

${ }^{51}$ Por ejemplo, $E s^{1}$ fol. 58 r, $E s^{2}$ fol. $69 \mathrm{v}$, B. fol. 60 r.

52 Por ejemplo, cuando Ŷābir b. Aflah calcula la magnitud del eclipse solar a partir de la latitud aparente de la Luna en la conjunción aparente evitando utilizar tablas. Cf. infra n. 78.

53 Lorch, "The Astronomical Instruments".

54 Lorch, "The Astronomy of Jābir b. Aflāḥ", 94.

Al-Qanțara (AQ) XXX 1, enero-junio 2009, pp. 83-136 ISSN 0211-3589 
de reescribir todo el Almagesto, tiene sentido también reelaborar este contenido.

El segundo criterio de edición es el de subsanar las deficiencias que puedan encontrarse en el Almagesto. Estas deficiencias no deben ser confundidas con errores.

En primer lugar, en numerosas ocasiones, Ptolomeo aproxima diversas demostraciones mediante trigonometría plana en lugar de utilizar trigonometría esférica, pues el teorema de Menelao dificultaba la aplicación de la misma. En estos casos, Ŷābir b. Aflah aplica trigonometría esférica basándose sobre todo en la regla de las cuatro cantidades y el teorema del seno. En estos casos, el procedimiento no varía desde un punto de vista astronómico respecto al dado por Ptolomeo, pero sí su solución geométrica.

Por otra parte, una de las deficiencias más importantes en el Almagesto es que, como bien señala Ŷābir b. Aflah, Ptolomeo es muy sintético en algunas ocasiones. A veces, delinea a vuelapluma un procedimiento sin proporcionar una figura en la cual apoyarse y en el que abusa de anáforas. El resultado es que el lector no sabe muy bien qué está diciendo ${ }^{55}$. En otras ocasiones, Ptolomeo enuncia una proposición sin demostrarla. Así pues, Ŷābir b. Aflah se propone llenar estos huecos apoyando sus demostraciones y procedimientos en las figuras necesarias y demostrando aquellas proposiciones únicamente enunciadas $(\$ 12)$.

Ŷābir b. Aflah llama la atención en su introducción al Iṣlāh al-Maŷisți sobre un caso particular dentro del criterio de edición que nos ocupa, consistente en subsanar las deficiencias del Almagesto. El texto de la introducción dice así:

También mencionó [Ptolomeo] que el centro de los deferentes de los tres planetas superiores divide en dos mitades la línea que va del centro del ecuante (markaz al-haraka al-mustawiya) al centro de la eclíptica (falak al-burūŷ). Éste es uno de los casos que admitió [acríticamente] pues no encontró una vía para acceder a este conocimiento mediante una demostración. Se nos ocurrió, gracias a la ayuda de Dios, sea bendito y ensalzado, una vía para acceder a este conocimiento mediante una demostración correcta $(\$ 10)$.

Es decir, Ȳābir b. Aflah llama la atención sobre el hecho de que Ptolomeo ha enunciado la necesidad de la bisección de la excentricidad

55 Por ejemplo, en el cálculo de la conjunción aparente a partir de la verdadera en el caso del eclipse solar donde interviene la paralaje lunar. Cf. PtA, 310-311.

Al-Qanțara (AQ) XXX 1, enero-junio 2009, pp. 83-136 ISSN 0211-3589 
en el caso de los planetas superiores, pero sin embargo no ha dado su demostración. La nueva edición del Almagesto subsana este defecto ${ }^{56}$.

El tercer criterio de edición, según nuestra relación, consiste en corregir las incoherencias que pudieran encontrarse en el texto tal como lo recibió Ŷābir b. Aflah en su traducción al árabe. Debe hacerse notar que estas incoherencias pueden ser debidas a la pluma de Ptolomeo o bien, tal como señala en su introducción, pueden tener su origen en problemas de traducción.

En cuanto a este último motivo, debe recordarse que el griego de Ptolomeo es difícil, especialmente por sus construcciones sintácticas ${ }^{57}$. Este hecho repercute en la labor de los traductores, y si se suma a las deficiencias en la exposición de Ptolomeo que acabamos de señalar, las traducciones resultantes podían ser cuando menos confusas. Ŷābir b. Aflah se propone redactar una obra original en árabe que, al no tener que seguir la literalidad del texto griego, facilite su comprensión. Sin embargo, en algunas ocasiones, el texto del Ișlāh al-Maŷisți copia literalmente la traducción del Almagesto de Ishāq b. Hunayn ${ }^{58}$.

Ahondando en este sentido, si bien Ŷābir b. Aflaḥ no lo indica, debemos tener en consideración los errores introducidos por el copista del manuscrito del Almagesto que maneja nuestro autor. Algunos de los errores que Ŷābir b. Aflah imputa a Ptolomeo son, de hecho, omisiones del copista del manuscrito. En estos casos, la corrección que sugiere Ŷābir b. Aflah puede no diferenciarse apenas de la solución dada por Ptolomeo. Ŷāair b. Aflah era consciente de que el texto del Almagesto con el que trabajaba no era del todo correcto. Sin embargo, achaca los errores al traductor, cuando probablemente el copista de su versión del Almagesto haya tenido un efecto mayor ${ }^{59}$. En este senti-

${ }^{56}$ En torno a esta demostración, vid. Swerdlow, "Jābir ibn Aflah", y Hugonnard-Roche, "La théorie astronomique".

57 En torno al estilo de Ptolomeo, en la introducción a la edición del Tetrabiblos, F.E. Robins señala: "Ptolemy, however, was a difficult author even for the ancients; the existence of the Paraphrase and the frequent flounderings of the anonymous commentator testify to this. He displays a certain enthusiasm for his subject, but beyond this it would be impossible to commend his literary style or even the clearness of his exposition. He is fond of long, involved sentences and has a number of mannerisms...". Cf. Robins, F.E., Ptolemy, Tetrabiblos. Edited and translated by F.E. Robbins, Cambridge (MA)-Londres, 1940, xxiv.

58 Por ejemplo, Ŷābir b. Aflah copia la traducción de Ishāq b. Hunayn de Almagesto I.2 y I.3 en el Libro II del Ișlāh al-Maŷisți. Cf. Ms. Es ${ }^{1}$ fols. 16v-17v.

59 Por ejemplo, la crítica de Ŷābir b. Aflah al cálculo de los eclipses lunares se debe a un error en el manuscrito del Almagesto que maneja. 
do, en un fragmento que se ha hecho famoso ${ }^{60}, \hat{Y} a \overline{b i r} b$. Aflah señala que ha consultado las dos traducciones principales del Almagesto —la de al-Haŷŷâŷ y la de Ishạa b. Hunayn - sin encontrar diferencias notables al respecto. Ȳābir b. Aflah señala:

Sin embargo, hemos investigado este punto en muchos manuscritos de la traducción de Hunayn y de la traducción de al-Ḥaŷŷâŷ sin encontrar más que escasas diferencias en la expresión. Ahora bien, en cuanto al significado no hemos encontrado ninguna diferencia en absoluto ${ }^{61}$.

Este comentario ha dado pie a subrayar una sensibilidad filológica en Ŷābir b. Aflạ̣ ${ }^{62}$. Sin embargo, nuestra investigación muestra que algunos de los errores que adscribe a Ptolomeo sólo se entienden si consideramos que el manuscrito del Almagesto con el que trabajaba estaba en algunos casos muy deteriorado. Por lo tanto, cabe sospechar que Ŷābir b. Aflah no siempre cotejó ambas traducciones del texto al menos en estos casos, ni tampoco varios manuscritos de la misma traducción. Sin embargo, ello no quiere decir que no utilizara ambas traducciones en alguna ocasión. Por una parte, Ŷābir b. Aflạ cita siempre la traducción de Ishạa b. Hunayn; pero por otra, el Ms. Berlín 5653 da el valor del período del mes sinódico lunar corregido por al-Haŷȳâŷ, lo que implica que consultó su traducción ${ }^{63}$.

Por último, dentro del tercer criterio de edición de nuestra relación, debemos señalar que principalmente Ŷābir b. Aflah se propone corregir las incoherencias salidas de la pluma del propio Ptolomeo respecto a las premisas en las que se basaba (§11). Al final de su Introducción, nuestro autor enumera diversas incoherencias que Ptolomeo comete a lo largo del Almagesto (§14). La finalidad de enumerarlas en su Introducción es facilitar su localización en el Ișlāh al-Maŷisțī a los lectores avanzados de su obra. Así pues, el Ișlāh al-Maŷisți está dirigido a dos públicos bien diferenciados: en primer lugar, estudiantes de astronomía neófitos para los que simplifica y subsana las deficiencias del Almages-

${ }^{60}$ Cf. Lorch, "The Astronomy of Jābir b. Aflāh", 96-97 y n. 61.

${ }^{61} \mathrm{Cf} \mathrm{Es}^{1}$ fol. $50 \mathrm{v}, \mathrm{Es}^{2}$ fol. 58r, B. fol. 50r.

${ }^{62}$ Cf. Carmody, Al-Bitruijii, 31-32.

${ }^{63}$ Cf. Bellver, "Jābir b. Aflah on the four-eclipse method", 186. Agradezco al Prof. Saliba que me aclarara las implicaciones de este dato. En torno a la corrección introducida por al-Haŷŷây, cf. Mancha, J.L., "A note on Copernicus' "correction' of Ptolemy's mean synodic month", Suhayl, 3 (2002-2003), 221-230.

Al-Qanțara (AQ) XXX 1, enero-junio 2009, pp. 83-136 ISSN 0211-3589 
to; y lectores avanzados a los que presenta sus correcciones teóricas en el seno de la astronomía ptolemaica.

Ŷābir b. Aflah, probablemente un autor poco o nada conocido en el momento de redactar el Ișlāh al-Maŷisțì, consciente de su atrevimiento al corregir a Ptolomeo, invoca la obligación de buscar la verdad frente al argumento de autoridad, y por tanto la obligación de corregir a Ptolomeo donde se considere necesario (§13). En este sentido, se escuda en una famosa paráfrasis de la Etica nicomaquea ${ }^{64}$ en la que Aristóteles confronta la verdad y el argumento de autoridad. Ŷābir b. Aflah señala que «cuando la verdad y Platón discuten, si bien ambos me son queridos, la verdad me es más querida». Es interesante subrayar el paralelo que establece Ŷābir b. Aflạ entre Ptolomeo y Platón, por una parte, y Aristóteles y su persona, por otra.

En cualquier caso, si bien Ŷābir b. Aflah se atreve a corregir a Ptolomeo, reclama para sí el mismo tratamiento que él da a Ptolomeo, a saber:

si nuestro parecer [sobre estos puntos] es verdadero, se adquirirá ciencia y nosotros recompensa; y si es erróneo, debe vencer la verdad, se nos recusará y se nos salvará de la oscuridad de la ignorancia, adquiriremos ciencia y se adquirirá recompensa y favor $(\S 13)$.

Esta actitud, plenamente científica por otra parte, parece indicar que Ŷābir b. Aflạ no había sometido a crítica todavía sus conclusiones y que es ahora, al presentar esta obra, cuando pretende darlas a conocer y que sean falsadas. Esto parece indicar que en su círculo más próximo no hay astrónomos competentes. Esta afirmación debe contraponerse con la indicación dada por Maimónides a propósito de la ubicación de Venus y Mercurio por encima del Sol, según la cual «grupos de andalusíes expertos en matemáticas» defendían esta tesis ${ }^{65}$.

Ŷābir b. Aflạ enumera quince errores en su Introducción al Ișlāh al-Mayisțti (§14). Sin embargo, no todos los errores que indica se encuentran reseñados en el índice de su Introducción, pues a lo largo del texto se localizan alusiones a errores menores que no se hallan reflejados en el mismo. En otros casos, varios de los errores que indica

64 Esta cita, según la da Ŷābir b. Aflah, “ تخاصم الحقت وأفلاطون وكلاهما حظي والحتّ الحظى منه " se " se inspira en Aristóteles, Ética a Nicómaco, I, 6, 1096a: 15-16; ed. Gredos, 1985, 135.

${ }^{65}$ Cf. supra n. 11.

Al-Qanțara (AQ) XXX 1, enero-junio 2009, pp. 83-136 ISSN 0211-3589 
pueden encontrarse detrás de una única crítica. Así, por ejemplo, la crítica en torno a la ubicación de Mercurio y Venus respecto al Sol y la Luna se extiende a lo largo de dos errores enumerados en el índice. Por otra parte, en algún caso, Ŷābir b. Aflạ critica las premisas que dan lugar a un capítulo del Almagesto, al ser incongruentes con hipótesis establecidas en algún otro lugar de la obra, como es el caso del Libro VIII donde estudia las estaciones y retrogradaciones de los planetas. Aduce que Ptolomeo establece dos anomalías en los modelos planetarios, pero, en cambio, cuando los aplica al estudio de sus estaciones y retrogradaciones, únicamente considera una anomalía. Ŷābir b. Aflah aplica las dos anomalías al estudio de sus estaciones y retrogradaciones, lo que le da pie a reescribir el libro XII del Almagesto. En definitiva, el índice de errores que aparece en la Introducción sirve como primer paso para el conocimiento de las críticas vertidas en el Ișlāh al-Maŷisțī. Sin embargo, un conocimiento cabal implicará el cotejo completo de ambas obras.

En definitiva, éstos son los tres criterios de edición que, a nuestro juicio, aplica Ŷābir b. Aflạ̣ en su redacción del Ișlāh al-Maŷisțī. Para comprender el papel de esta obra en la historia de la Astronomía, especialmente en el mundo latino, es necesario atender a estos tres criterios en conjunto, pues en otro caso podemos tener una visión sesgada de la misma. La intención principal del autor, lo hemos visto, es facilitar el estudio del Almagesto simplificando su matemática y cubriendo los vacíos que pudiera haber dejado Ptolomeo, para lo que introduce la nueva trigonometría esférica que tomarán los latinos. La intención principal del autor es, pues, redactar el Almagesto en forma de texto docente, en la forma de unos Elementos de Astronomía. Por ello, algunos de los títulos que recibe esta obra son Kitāb al-Hay'a, Liber de Astronomia o Elementa Astronomica. La intención secundaria del autor es corregir algunas incoherencias que aparecen en el Almagesto derivadas de las premisas que asume Ptolomeo. Cuando atendemos a estas dos intencionalidades en conjunto, cobran sentido los títulos Ișlāh al-Hay'a e Ișlāḥ al-Maŷisțī. Pese al eco que recibieron algunas de sus correcciones, no debemos perder de vista que Ŷābir b. Aflah redacta básicamente unos elementos de astronomía matemática y que el Ișlāh al-Maŷisți fue leído así en la mayoría de los casos hasta el s. XVII. Si éstas son las dos finalidades de la obra, un resultado accidental fue la difusión de la nueva trigonometría esférica en Occidente. A este resultado alude la catalogación del Ms. Escorial 
910 del Isslāh al-Maŷisțī como Abu Mohamed Giaber Sivilianiy de Sphera, de Eclypsi lunari ac solari ${ }^{66}$.

\section{Correcciones al Almagesto}

En primer lugar, Ŷābir b. Aflah indica que Ptolomeo cometió un error (wahm) en Almagesto IV.2 donde trata el método de Hiparco para obtener el período de la anomalía lunar basándose en dos pares de eclipses lunares $(\S 14.1)^{67}$. Si el movimiento de la Luna en dos eclipses es similar, es posible que la anomalía de la Luna sea la misma en ambos casos. Si la duración de dos intervalos determinados, cada uno, por dos eclipses es el mismo, podemos estar ante intervalos temporales que incluyan un número entero de retornos de la Luna en su anomalía. Ptolomeo da unas condiciones para tener la certeza de obtener el período de la anomalía lunar e introduce una casuística adicional que debe tenerse en cuenta. Ŷābir b. Aflah añade dos condiciones adicionales relativas a la velocidad lunar con lo que hace innecesaria la casuística descrita por Ptolomeo ${ }^{68}$. Pese a que el método de Ptolomeo es correcto, la propuesta de Ŷābir b. Aflah es mucho más simple y elegante.

Ŷābir b. Aflah señala a continuación un error en Almagesto V.10 ${ }^{69}$ cuando, a propósito del modelo lunar, estudia si la influencia que ejerce la excentricidad del deferente es significativa en las sizigias (§14.2). Para ello, Ptolomeo analiza los casos en los que, cuando el Sol y la Luna se encuentren en conjunción u oposición verdadera, las anomalías son máximas. Si bien el método de Ptolomeo expuesto en su demostración es correcto, Ŷābir b. Aflạ señala que el enunciado que la introduce difiere de la demostración en sí ${ }^{70}$. Esta discrepancia le lleva a consultar diversos manuscritos de las traducciones de al-Ḥaŷȳâŷ y de Isḥāq b. Ḥunayn en busca de un posible error textual cometido por el traductor ${ }^{71}$.

${ }^{66}$ Cf. la descripción del Ms. 905, ahora Ms. Escorial 910, en Casiri, M., Biblioteca Arabico-Hispana Escurialensis, Madrid, 1760, I, 345.

${ }^{67}$ Cf. PtA, 174-179 y Neugebauer, O., A History of Ancient Mathematical Astronomy, Berlín-Heidelberg-Nueva York, 1975 (en adelante, HAMA), 71-73.

${ }^{68}$ Cf. Bellver, "Jābir b. Aflah on the four-eclipse method".

${ }^{69}$ Cf. PtA, 239-243 y HAMA, 98-99 y 1234.

${ }^{70}$ Cf. PtA, 240.

71 Cf. supra n. 61.

Al-Qantara (AQ) XXX 1, enero-junio 2009, pp. 83-136 ISSN 0211-3589 
El siguiente error se encuentra en la delimitación de los límites máximos de los eclipses $(\S 14.3)^{72}$. Ptolomeo desea conocer los argumentos en latitud máximos en los que sea posible que se produzca un eclipse solar o lunar a partir de la localización de la sicigia media en el orbe inclinado de la Luna. En la obtención de la sicigia verdadera del eclipse solar — un paso intermedio - , Yābir b. Aflaḥ ${ }^{73}$ señala que Ptolomeo ha cometido el error de confundir la posición verdadera de la Luna en el instante de la sicigia aparente con su posición en la sicigia verdadera ${ }^{74}$. Ŷābir b. Aflah soluciona este error hallando correctamente la posición de la sicigia verdadera.

El siguiente error que señala Ŷābir b. Aflah se encuentra en la descripción del método de Ptolomeo para el cálculo de la magnitud y las fases de los eclipses $(\$ 14.4)^{75}$. Este método es común para eclipses lunares y solares, si bien el eclipse solar requiere el tratamiento adicional del efecto de la paralaje lunar. Ŷābir b. Aflạ̣ señala que se halla un error en el eclipse lunar, pero esta indicación es de hecho aplicable también al eclipse solar. Ptolomeo adopta una serie de premisas, como el uso de geometría plana y ciertas aproximaciones que Ŷābir b. Aflah critica. Asimismo, Ptolomeo calcula dos tablas para casos particulares: cuando la Luna se sitúa en el apogeo y cuando se sitúa en el perigeo. Para cualquier otra anomalía lunar, Ptolomeo utiliza una tabla para interpolar los valores obtenidos en las dos situaciones previas. Ȳābir b. Aflah evita el uso de interpolación y de tablas aportando un método completamente diferente del de Ptolomeo ${ }^{76}$. Por otra parte, indica la existencia de un error en el método de interpolación que aplica Ptolomeo ${ }^{77}$. Sin embargo, el método de Ptolomeo en este caso no se corresponde al que le adscribe Ŷābir b. Aflaḥ. Gracias a la cita que da Ŷābir b. Aflah de este pasaje del Almagesto, se concluye que el autor del Ișlāh al-Maŷisțī se basa en un texto corrupto, por lo que la crítica que le dedica es injustificada.

Tras el error anterior que podría aplicarse a cualquier tipo de eclipse, Ŷābir b. Aflaḥ señala la existencia de errores adicionales en el tra-

72 Cf. PtA, 239-243, у HAMA, 98-99 у 1234.

${ }^{73}$ Cf. Mss. Es ${ }^{1}$ fols. $50 r-51$ r, Es ${ }^{2}$ fols. $57 r-58 v$ y B. fols. $49 v-50 v$.

74 Cf. PtA, 286.

75 Cf. PtA, 294-310; HAMA, 134-139 y 1240, y Pedersen, O., A Survey of the Almagest, Odense, 1974, 231-235 para el cálculo de eclipses lunares y solares.

${ }_{76}$ En torno a este método, cf. Bellver, "Jābir b. Aflah on lunar eclipses".

77 Cf. Mss. Es ${ }^{1}$ fols. 62r-64r, Es ${ }^{2}$ fols. $74 v-77$ r y B. fols. 64r-66r. 
tamiento ptolemaico del eclipse solar $(\S 14.5$ y $§ 14.6){ }^{78}$. El eclipse solar se diferencia del lunar por el efecto de la paralaje. En la descripción del tratamiento de la paralaje, el procedimiento propuesto por Ŷābir b. Aflah sigue de cerca el procedimiento de Ptolomeo. Resumiremos brevemente los errores siguiendo el orden del texto ptolemaico.

Ŷābir b. Aflạ señala, a propósito de la obtención de la longitud de la conjunción aparente a partir de la verdadera, que Ptolomeo toma como referencia, para sumar o restar los tiempos determinados por el efecto de la paralaje y la epiparalaje, el meridiano en la conjunción verdadera (§14.5). Sin embargo, aduce, este signo debe ser función de la longitud de la conjunción verdadera respecto a la longitud del medio cielo del ascendente en la conjunción verdadera. Ahora bien, Ptolomeo toma como referencia el medio cielo del ascendente y no el meridiano ${ }^{79}$, como erróneamente le atribuye Ŷābir b. Aflaḥ. Así pues, nos encontramos ante un error de lectura o un error en la transmisión del texto del Almagesto con el que trabaja Ŷābir b. Aflah.

En el siguiente error, Ŷābir b. Aflah alude al punto en el que Ptolomeo resuelve el argumento en latitud aparente para obtener, a continuación, la magnitud y la duración de las fases del eclipse solar (§14.6). La objeción que plantea Ŷābir b. Aflaḥ es confusa, y más teniendo en cuenta que el método de Ptolomeo es correcto si hacemos caso omiso de su aproximación con trigonometría plana. Ŷābir b. Aflah cita el texto del Almagesto en el que apoya su crítica. La comparación de esta cita con las traducciones árabes del Almagesto muestra claramente que hay una corrupción en el manuscrito del Almagesto con el que trabaja Ŷābir b. Aflah. En ultima instancia, la corrección de Ŷābir b. Aflah es equivalente al método de Ptolomeo, con lo que la crítica que le hace es de nuevo injustificada.

Por último, el tercer error se encuentra en el estudio que dedica Ptolomeo a la diferente duración de la fase de inmersión y emersión del eclipse solar en función de la paralaje lunar. Este error no se encuentra reseñado en la lista de errores de la Introducción al Ișlāh al-Maŷisți. Ptolomeo señala que si el instante medio del eclipse coincide con el tránsito solar por el meridiano del lugar, las fases de in-

${ }^{78}$ Para el conjunto de estos tres errores, cf. Mss. Es ${ }^{1}$ fols. $64 \mathrm{v}-67 \mathrm{r}, \mathrm{Es}^{2}$ fols. $78 \mathrm{r}-80 \mathrm{v}$ y B. fols. $66 \mathrm{v}-68 \mathrm{v}$.

${ }^{79}$ Cf. PtA, 311.

Al-Qantara (AQ) XXX 1, enero-junio 2009, pp. 83-136 ISSN 0211-3589 
mersión y emersión son iguales, pues tiene en cuenta el movimiento de la eclíptica en el horizonte. En cambio, Ȳābir b. Aflah aduce que la fase de inmersión y emersión son iguales cuando el instante medio del eclipse tiene lugar en el medio cielo del ascendente, pues tiene en cuenta el movimiento de la Luna en su orbe inclinado. De hecho, ambos factores influyen, por lo que la crítica planteada por Ŷābir b. Aflah es correcta, pero no, en cambio, su solución.

El cotejo de estas tres críticas al tratamiento ptolemaico del eclipse solar arroja un resultado escaso. Sin embargo, sería injusto desdeñar su valor, pues muestra que Ŷābir b. Aflah entiende los procedimientos ptolemaicos y que es capaz de restituirlos cuando una corrupción textual impide la correcta transmisión. Además, quizá el verdadero valor de este capítulo se encuentre en su capacidad para aclarar el, por otra parte, muy confuso tratamiento ptolemaico del efecto de la paralaje lunar en el eclipse solar.

La última crítica en el libro V del Ișlāh al-Maŷisțī a la teoría de los eclipses en el Almagesto se centra en la inclinación de los eclipses o prosneusis. Este concepto había sido utilizado en la astronomía preptolemaica con el objeto de elaborar predicciones astrometeorológicas ${ }^{80}$. Esta crítica no aparece en el índice de críticas de la Introducción al Ișlạh. Quizá el motivo de que no aparezca es que no se trata propiamente de una corrección de carácter teórico, sino más bien de una reelaboración de un método aplicando un procedimiento basado en geometría esférica más sólido que el que sigue Ptolomeo quien había simplificado sobremanera su aproximación. Si bien, la crítica es completamente válida desde un punto de vista teórico, Ŷābir b. Aflah no parece tener en cuenta la intencionalidad y el grado de exactitud que pretende Ptolomeo con su método, quien, por otra parte, era plenamente consciente de que su aproximación era, por lo menos, grosera ${ }^{81}$.

El resto de críticas que aparecen enumeradas en el índice del Ișlāh al-Maŷisți se centra en la teoría de los planetas. La primera de ellas es, quizá, la que ha tenido una repercusión más importante en autores medievales posteriores ${ }^{82}$. Al comienzo del Libro VII del Iṣlāḥ

${ }^{80}$ Almagesto VI.11 y VI.13.

${ }^{81}$ Cf. Bellver, "Ŷābir b. Aflah en torno a la inclinación".

${ }^{82}$ En torno a un muy breve resumen de la misma en el que se ha basado la historiografía posterior, cf. Delambre, Histoire de l'Astronomie, 184. 
al-Maŷisțī ${ }^{83}$, que corresponde al comienzo del Libro IX del Almagesto ${ }^{84}, \hat{Y} \bar{a} b i r$ b. Aflah se plantea el orden de las esferas asociadas a los planetas ( $\$ 14.7$ y $\S 14.8)$. Ptolomeo no concede mucha importancia a este problema, pues no le dedica apenas espacio en el Almagesto y toma el orden tradicional de los planetas casi sin justificarlo. La tesis de Ptolomeo, siguiendo a los primeros astrónomos, es que las esferas de Venus y Mercurio se sitúan por debajo del Sol ${ }^{85}$. Sin embargo, algunos astrónomos posteriores habían mantenido que las esferas de Mercurio y Venus debían de situarse por encima de la del Sol ${ }^{86}$. Las razones que da Ptolomeo en defensa de su tesis son vagas. En primer lugar, considera que es posible que no se produzcan tránsitos solares de Venus y Mercurio. A continuación, apoyándose en la afirmación de que los astros — léase estrellas y planetas - no tienen paralaje, señala que no es posible calcular sus distancias. Así pues, para justificar el orden dado, aduce que se conforma más al orden natural (amr tabi $\left.\bar{l}^{`} \hat{\imath}\right)$ de las cosas que el Sol se halle por encima de Venus, pues de este modo se separan los planetas que tienen elongación limitada respecto al Sol - Mercurio y Venus - de los que no la tienen - Marte, Júpiter y Saturno-.

Ŷābir b. Aflah ${ }^{87}$, en cambio, objeta que, según Ptolomeo, el Sol tiene una paralaje sensible ${ }^{88}$, con lo que es posible calcular su distancia geocéntrica (\$14.7). Por otra parte, tras una demostración geométrica, muestra la necesidad de la existencia de puntos de intersección entre los epiciclos de Venus y Mercurio y la eclíptica, con lo que es necesario que se produzcan tránsitos solares de Venus y Mercurio en el caso de que sus esferas se hallen por debajo de la del Sol (§14.8).

83 Ms. Es ${ }^{1}$ fols. $78 v-81$ r.

84 PtA, 419-420.

85 Para una relación de las diversos posibles órdenes de los planetas entre los antiguos, cf. HAMA, II, 690-693. Neugebauer enumera entre los que apoyaban esta tesis: Platón, Eratóstenes y Arquímedes. Los seguidores de ambos órdenes han sido agrupados en dos escuelas: los pitagóricos consideraban que las esferas de Venus y Mercurio se encontraban debajo de la del Sol y los platónicos, encima. En torno al orden de las esferas en Arquímedes, cf. Osborne, C., "Archimedes on the Dimensions of the Cosmos", Isis, 74, 2 (1983), 234-242. Osborne, a diferencia de Neugebauer, considera improbable que Arquímedes situara Venus y Mercurio por encima del Sol. En lugar de ello, apoya la tesis de que Arquímedes parte de un orden de origen pitagórico.

86 Dreyer, J.L.E., A History of Astronomy from Thales to Kepler, Nueva York, 1953 (1. ${ }^{a}$ ed.: History of the Planetary Systems from Thales to Kepler, Cambridge, 1906), 168.

$87 \mathrm{Ms} . \mathrm{Es}^{1}$ fol. $78 \mathrm{v}$.

88 Cf. Almagesto V.17 (PtA, 258-264).

Al-Qanțara (AQ) XXX 1, enero-junio 2009, pp. 83-136 ISSN 0211-3589 
Como ello no es así, debe concluirse que sus esferas se hallan por encima de la del Sol.

En cuanto al orden natural, Ŷābir b. Aflah señala que el Sol y la Luna tienen características comunes que los diferencian de los planetas. En este sentido es más acorde al orden natural agruparlos según la similitud de sus características: los dos luminares - Sol y Luna-, por una parte, y los planetas por otra.

La siguiente crítica de Ŷābir b. Aflah se centra en el método para determinar los apogeos de los planetas inferiores (§14.9 y §14.10). Se encuentra, como la crítica anterior en el libro VII del Ișlāh al-Maŷisțī y corresponde a Almagesto IX.6. Ŷābir b. Aflah critica a Ptolomeo al determinar el apogeo de Mercurio quien, habiendo probado que dos elongaciones máximas y opuestas de Mercurio respecto al Sol medio y simétricas respecto al apogeo deben ser iguales, considera que el argumento inverso también será cierto. Sin embargo, Ŷābir b. Aflah muestra que ello no es así y da una solución alternativa (§14.9) ${ }^{89}$.

La última crítica del Libro VIII del Ișlāh al-Maŷisțī se centra en la cuarta figura de Almagesto IX.6 ${ }^{90}$. Y̌ābir b. Aflah señala que Ptolomeo comete el error de situar el centro del deferente de Mercurio en el lado equivocado de la línea tendida entre el apogeo y perigeo con lo que da radios erróneos del deferente excéntrico ${ }^{91}$. Pese a ello, este error no tiene implicaciones en la demostración de Ptolomeo $(\S 14.10)$.

En el libro VIII del Iṣlāh al-Maŷisțī, Ŷābir b. Aflah se propone estudiar las estaciones y retrogradaciones de los planetas atendiendo a las dos anomalías y señala cuatro errores en esta sección del Almagesto ${ }^{92}$.

En el primer error de los cuatro, Ŷābir b. Aflạ señala que Ptolomeo considera que el punto de estación de un planeta coincide con aquel punto del epiciclo que hace que, tomando como punto de referencia el centro de la eclíptica, la razón del segmento interior al epiciclo respecto al exterior equivale a la razón del movimiento aparente

89 Cf. Samsó, "Ibn al-Haytham".

${ }^{90}$ Cf. PtA, 447, fig. 9.4.

91 En torno a este error, cf. Samsó, "Ibn al-Haytham", 215.

92 Cf. PtA, 555-562, y HAMA, 190-206 y 1168-1172, para el tratamiento de las estaciones y retrogradaciones de los planetas en general. Pese a que el modelo final de los planetas consta de dos anomalías, Ptolomeo basó su cálculo de las estaciones y retrogradaciones de los planetas en función de una única anomalía.

Al-Qanțara (AQ) XXX 1, enero-junio 2009, pp. 83-136 ISSN 0211-3589 
en longitud respecto al movimiento aparente, es decir respecto al movimiento del planeta en su epiciclo tras tener en cuenta el ángulo de la anomalía debido a la excentricidad ${ }^{93}$. Sin embargo, Ŷābir b. Aflah considera que el punto de estación coincide con el punto del epiciclo que divide la línea que sale del centro de la eclíptica según la proporción del movimiento aparente en longitud respecto al movimiento en anomalía, no respecto al movimiento aparente (§14.11).

A continuación, Ŷābir b. Aflah señala que Ptolomeo comete otro error al obtener la distancia de los puntos de estación respecto al perigeo aparente (§14.12) ${ }^{94}$. El autor del Almagesto considera que la distancia de los puntos de estación depende de la variación de la distancia del centro del epiciclo respecto al centro de la eclíptica. Ŷābir b. Aflah menciona que es posible que esta distancia varíe sin que se produzca una variación en la distancia de los puntos de estación respecto al perigeo aparente. Propone, en cambio, que únicamente se tengan en cuenta las razones de líneas trazadas desde el centro de la eclíptica.

Ŷābir b. Aflạ señala que Ptolomeo comete un tercer error pues obtiene la segunda estación doblando el arco entre la primera estación y el perigeo aparente $(\S 14.13){ }^{95}$. Esto sólo es así si no se tiene en cuenta la segunda anomalía de los planetas. Ŷābir b. Aflah, al tenerla en cuenta, concluye que la afirmación de Ptolomeo sólo es correcta si el centro del epiciclo se halla en uno de los mesogeos ${ }^{96}$ de la excéntrica.

El último error que atribuye a Ptolomeo en el Libro VIII consiste simplemente en el cómputo del efecto del error anterior en los casos de Venus y Marte (\$14.14) ${ }^{97}$. Las diferencias entre los períodos de retrogradación que da Ŷābir b. Aflah respecto a los dados por Ptolomeo ascienden a un máximo de 18 días, para el caso de Marte, y de dos días y medio, para el caso de Venus.

La última crítica que aparece en el índice de críticas de la introducción remite al libro IX del Ișlāh al-Maŷisțī dedicado al estudio de las latitudes planetarias (§14.15). Ŷābir b. Aflah se centra en el tratamiento de la «inclinación» de Venus y Mercurio que se encuentra en el capítulo XIII.4 del Almagesto. Ptolomeo considera que, para el

93 Cf. Mss. $\mathrm{Es}^{1}$ fol. 102 r, $\mathrm{Es}^{2}$ fol. $121 \mathrm{r}$ y B. fol. $101 \mathrm{v}$.

${ }^{94}$ Cf. Mss. $\mathrm{Es}^{1}$ fol. $104 \mathrm{r}, \mathrm{Es}^{2}$ fol. $123 \mathrm{r}$ y B. fol. 103v.

${ }^{95}$ Cf. Mss. Es ${ }^{1}$ fol. 104v, Es ${ }^{2}$ fol. 124r y B. fol. 104r.

96 Entiendo por "mesogeo" el punto de un epiciclo o una excéntrica en el que se iguala el movimiento verdadero del astro a su movimiento medio.

${ }^{97}$ Cf. Mss. $\mathrm{Es}^{1}$ fol. 105 r, $\mathrm{Es}^{2}$ fol. $124 \mathrm{v}$ y B. fol. $104 \mathrm{v}$. 
cálculo de la inclinación, puede utilizar una función auxiliar cuya forma coincide con la ecuación del argumento del modelo en longitud. Así pues, Ptolomeo puede utilizar con este fin la ecuación del argumento si bien corregida. Ptolomeo considera que la corrección a la ecuación del argumento es máxima cuando la elongación es máxima ${ }^{98}$, lo cual no es correcto. Ŷābir b. Aflah corrige la afirmación de Ptolomeo señalando que esto sólo es así en el caso de Mercurio, pero no en el caso de Venus ${ }^{99}$.

En conclusión, la Introducción del Ișlāh al-Maŷisțì muestra que la intención de Ŷābir b. Aflaḥ en la redacción de su obra es básicamente facilitar el acceso al Almagesto y no reescribir una nueva astronomía. Por otra parte, el análisis de las críticas que aparecen en el Ișlāh al-Maŷisțī muestra que éstas son de carácter técnico y que se derivan, en última instancia, del hecho de que la astronomía de Ptolomeo no sea totalmente coherente entre sí. Ŷābir b. Aflah busca un sistema ptolemaico totalmente coherente y no un nuevo sistema astronómico como en el caso de los filósofos del s. XII; es decir Ŷābir b. Aflaḥ es un astrónomo ultra-ptolemaico, no anti-ptolemaico. Sin embargo, pese a no pertenercer a la «rebelión andalusí contra la astronomía ptolemaica», el Ișlāh al-Maŷisțī será leído con atención por los filósofos de la segunda mitad del s. XII. El Iṣlāh al-Maŷisțī les servirá en dos sentidos. En primer lugar, estudiarán el Almagesto con el Ișlāh al-Maŷisți al lado ${ }^{100}$, apoyándose en él para comprender la obra de Ptolomeo respondiendo así a la finalidad primera de la obra. En segundo lugar, la crítica en torno al orden de los planetas no les pasará desapercibida. Probablemente, esta crítica y las objeciones de Ibn al-Zarqālluh en torno al movimiento de las estrellas fijas, según señala al-Bitrūŷȳ ${ }^{101}$, ambas producidas en el seno de la astronomía matemática, contribuyeron a rebajar el prestigio del sistema ptolemaico a ojos de estos autores permitiendo que aflorasen las críticas a Ptolomeo, pues la Naturaleza contradice sus hipótesis ${ }^{102}$. Así pues, Ŷābir b. Aflah, matemático ultra-ptolemaico, sirve como uno de los ele-

98 Cf. PtA, 624, y Pedersen, A Survey of the Almagest, 382.

${ }^{99}$ Mss. Es $^{1}$ fol. $112 \mathrm{v}, \mathrm{Es}^{2}$ fol. $133 \mathrm{v}, \mathrm{B} .111 \mathrm{v}$.

100 Lay, J. "L'Abrégé de l'Almageste: un inédit d'Averroès en version hébraïque", Arabic Sciences and Philosophy, 6, 1 (1996), 40.

101 Goldstein, B.R., Al-Bitrujiz: On the principles of Astronomy, New Haven-Londres, 1971, 1, 53.

102 Ibidem.

Al-Qanțara (AQ) XXX 1, enero-junio 2009, pp. 83-136 ISSN 0211-3589 
mentos catalizadores de una reacción anti-ptolemaica y filo-aristotélica ${ }^{103}$. Tenemos un ejemplo de estas dos posiciones casi antagónicas en la diferente actitud de Ŷābir b. Aflah y los filósofos del s. XII respecto al ecuante. Ŷābir b. Aflah critica la inconsistencia matemática de Ptolomeo al no demostrar a priori la bisección de la excentricidad en los planetas superiores, mientras que los filósofos del s. XII critican la propia existencia del ecuante en cuanto viola el postulado de que los movimientos celestes sean uniformes en torno al centro de la Tierra. Ȳābir b. Aflah tiene un lugar por derecho propio en la historia de las críticas a Ptolomeo, pero no podemos encuadrarlo junto con los filósofos del s. XII mencionados.

\section{Criterios de edición}

Esta edición, que no se pretende crítica pues no hemos utilizado los manuscritos de las traducciones latinas o hebreas ni los hebraico-árabes, se basa en los tres manuscritos árabes conservados en alfabeto árabe. Los tres manuscritos son: Escorial 910 abreviado como Es ${ }^{1}$; Escorial 930 abreviado como Es²; y Berlín 5653 abreviado como B.

Se han anotado las variantes de los diferentes manuscritos. Si en nota se da una variante, debe asumirse que la versión que aparece en la edición considerada correcta corresponde a los manuscritos que no aparecen en nota.

Se ha indicado el alcance de la nota en el texto considerado correcto mediante dos claudators. Por ejemplo,

$$
\text { قَال أبو محمّ جابر بن أفلح (الإشبيلي\{1 }
$$

En este caso, la palabra al-Išbīlī tiene alguna variante en alguno de los manuscritos. Podemos encontrar también claudators anidados. El tratamiento de los mismos es el equivalente al de una jerarquía de paréntesis en una expresión matemática. Por último, en el caso de que se encuentre una nota sin claudators en el texto árabe, la variante anotada no substituye ninguna palabra en la edición considerada correcta.

103 "The episode referred to in the title of this chapter as "the Andalusian revolt" is the well-known anti-Ptolemaic program of research that was conceived and defended by twelfth-century scholars in Muslim Spain"; cf. Sabra, "The Andalusian Revolt”, 133.

Al-Qanțara (AQ) XXX 1, enero-junio 2009, pp. 83-136 ISSN 0211-3589 


\section{Traducción}

[Es ${ }^{1}$ fol. $2 v, E s^{2}$ fol. 1v y B. fol. 1v]

[§1] En el nombre de Dios el Clemente el Misericordioso. La bendición de Dios y la paz sean sobre nuestro señor Muhammad y sobre su familia.

Dijo Abū Muhammad Ŷābir b. Aflah al-Išbīlī, Dios le tenga en Su misericordia:

La alabanza pertenece a Dios, el Primero sin principio y el Último sin fin, quien sin tener igual creó las cosas y las determinó por Su sabiduría con la mejor y más bella de las formas. La bendición de Dios sea sobre Su Profeta, quien trajo la buena nueva. Él es Su lámpara luminosa (sirāŷy munīr) ${ }^{104}$ con la que [Dios] guió a las criaturas (jalā'iq) y mediante la que manifestó las realidades (haq $\left.\bar{a}^{\prime} i q\right)$.

[§2] Dios, sea ensalzado, honró a la especie humana (naw' al-insān) y la antepuso a todos los animales por el intelecto ('aql). Dispuso [el intelecto] como una luz (nür) a la que se puede recurrir en las tinieblas (zulam) y por medio de la cual se aprende (ta 'allama) lo que no era conocido. Dijo, sea glorificada Su Majestad, «Creó al ser humano. Le enseñó ('allama) la explicación clara (al-bayān)» ${ }^{105}$. Y [Dios], sea bendito Su nombre, dirigiéndose a Muhammad, Su siervo y enviado, la bendición y la paz estén con él y con su familia, los inmejorables (al-tayyibūn), favoreciéndole y avisándole con Su gracia, dijo: «Y [Dios] te enseñó ( 'allama) [Oh Profeta] lo que no conocías, y el honor que Dios te concedió fue inmenso»" ${ }^{106}$.

[§3] La ciencia ( $\mathrm{llm}$ ) se divide en diversos tipos de saber. Tras la ciencia de la Ley Revelada ( 'ilm al-šarī'a), los saberes más nobles son aquellos cuyos contenidos son estables e imperecederos sin que sufran ningún cambio y [en los que se cumpla] que los métodos que dan acceso al conocimiento de [estos contenidos] garanticen la certeza y sean indudables de tal forma que conduzcan a su buscador a la verdad cierta.

[§4] La ciencia de la astronomía ('ilm al-hay’a, lit. «ciencia de la estructura») [que estudia] los movimientos del Sol, de la Luna, de las estrellas (nuŷūm) y sus esferas (aflāk) y las restantes cosas que la

\footnotetext{
104 Cf. Corán 33, 46.

105 Corán 55, 4.

106 Corán 4, 113.
}

Al-Qanțara (AQ) XXX 1, enero-junio 2009, pp. 83-136 ISSN 0211-3589 
acompañan es una ciencia que supera a la mayoría de las ciencias por reunir en sí las características que le conceden la superioridad, pues sus contenidos son estables e imperecederos sin que sufran ningún cambio, mientras Dios, sea ensalzado, se lo conceda, y [en la que se cumple] que los métodos que dan acceso al conocimiento de [estos contenidos] garantizan la certeza y son claros. Así pues [esta ciencia] obtuvo la superioridad dadas sus características.

[§5] Con Claudio Ptolomeo esta ciencia alcanzó la perfección pues reunió los conocimientos de los antiguos previos a él, a los que sumó lo que él mismo aprendió tras ellos. Escribió todo esto en su libro conocido con el nombre de Almagesto. Por ello nos legó una bendición generosa y de grandes proporciones. Su libro pasó a ser suficiente para el dominio de esta ciencia al reunir en sí todos los temas. Sin embargo el manejo para el estudiante es difícil por varios motivos:

- [§5.1] En primer lugar une la parte teórica y la práctica. Por ello, el procedimiento práctico obliga a multiplicar unos números por otros, a dividir unos por otros, a obtener sus raíces cuadradas y a elaborar [Es ${ }^{2}$ fol. $\left.2 \mathrm{r}\right]$ tablas dedicadas al ámbito práctico. Así pues, por esta razón el libro se alarga, el contenido teórico queda dividido y la mezcla con el contenido práctico dificulta la tarea del lector.

- [§5.2] Utiliza en muchas de sus demostraciones el teorema de Menelao (al-Šakl al-Qatțāa). Éste es un teorema difícil que se ramifica en multitud de formas y en el que es necesario el uso de razones compuestas, lo que resulta molesto, por lo que se dificulta al estudiante su control, su comprensión y [obtener] el resultado del mismo.

- [§5.3] Remite sus demostraciones al libro de Teodosio y al libro de Menelao y ambos son difíciles y abstrusos. El estudiante esforzado no estará preparado para detenerse en ellos y estudiar detenidamente ambos libros y el teorema de Menelao (al-Sakl al-Qatțā') hasta que transcurra al menos un año entero. Quizá después de esto puede caer en la pereza o se reducirá el tiempo para proseguir con el plan del libro.

- [§5.4] Resumió su discurso en numerosos lugares de su libro con lo que se dificulta [B. fol. 2r] su comprensión desconcertando grandemente al estudiante hasta el punto que esto llama a la pereza y a un exceso de aburrimiento.

- [§5.5] Con el paso de una lengua a otra que han operado los traductores del libro se produce una anteposición o posposición en [el 
orden de] la expresión y una distancia entre los significados, lo que confunde al lector y lo desconcierta. Esto provoca que tenga que dedicarse a un único tema un tiempo excesivo y que no pueda articular los significados buscados con el fin de comprender [Es ${ }^{1}$ fol. $\left.3 r\right]$ unos con otros. Así pues puede que con ello no persista suficientemente en el estudio.

[§6] Nos aconteció que dado el amor y la pasión por esta ciencia debido a las cosas que mencionamos y dado que, también, [Ptolomeo] había mencionado en el comienzo de su libro las razones que llevan a la pasión y el amor por ella no pudimos dejar de estudiarlo con atención y sobrellevamos las penalidades y dificultades que sufre su lector recurriendo a nuestra experiencia previa y seguridad en el arte de la geometría hasta que, gracias a Dios, sea bendito y ensalzado, comprendimos todo el contenido de este libro en torno a la astronomía ('ilm al-hay'a). Después de esto, no dejamos de persistir en el estudio y continuamos la investigación y la reflexión para que pudiera facilitarse [al estudiante satisfacer su] anhelo de esta ciencia enorme y acercar la comprensión de este libro inmenso.

[§7] Así pues, se nos ocurrió gracias a la ayuda de Dios, sea bendito y ensalzado, y a Su excelente asistencia y apoyo [añadir] unos contenidos introductorios fáciles y concisos con los que se permitiera prescindir del libro de Menelao, del teorema de Menelao (al-šakl al-qatțā ) y de la mayor parte del libro de [Es ${ }^{2}$ fol. $\left.2 \mathrm{v}\right]$ Teodosio, y con los que pudiéramos obtener la incógnita con los datos conocidos en astronomía mediante una proporción simple (mutanāsiba) de cuatro elementos y no con una proporción compuesta (mu'talafa) de seis elementos como en el caso del teorema de Menelao (al-šakl al-qațtā ). De esta forma se facilita la obtención de la incógnita a partir de los datos conocidos puesto que el número de variables conocidas necesarias es menor. Por este motivo el anhelo para conocerlo se hace más fácil [cumplirlo], disminuye la complicación y el esfuerzo.

[§8] Se nos ocurrió que, en estos contenidos introductorios que hemos mencionado, además de lo que señalamos en cuanto al número reducido de variables y al número escaso de veces que se tiene que recurrir a la proporción compuesta, pondríamos los medios para realizar todos los casos que se utilizan y sin que necesitemos utilizar líneas rectas y sus ángulos correspondientes, en lugar de los arcos y sus ángulos, 
como hacía Ptolomeo en algunos lugares de su libro pues no podía proceder de otra manera. Esto sin duda da lugar a aproximaciones.

[§9] [Ptolomeo] utilizó para las observaciones que necesitaba cuatro instrumentos con ocho armillas. Se nos ocurrió un único instrumento que no necesita sino una armilla, un cuadrante de otra y una regla que permite prescindir de todos los instrumentos que menciona.

[§10] También mencionó [Ptolomeo] que el centro de los deferentes de los tres planetas superiores divide en dos mitades la línea que va del centro del ecuante (markaz al-haraka al-mustawiya) al centro de la eclíptica (falak al-burūŷy). Éste es uno de los casos que admitió [acríticamente] pues no encontró una vía para acceder a este conocimiento mediante una demostración. Se nos ocurrió, gracias a la ayuda de Dios, sea bendito y ensalzado, una vía para acceder a este conocimiento mediante una demostración correcta.

[§11] Cuando miramos con atención, se nos hizo patente que le había alcanzado la limitación particular de la naturaleza del ser humano - sea glorificado el Único en la perfección y sea ensalzado $\mathrm{Su}$ nombre- - Y junto a sus limitaciones que mencionamos anteriormente, encontramos que había fantaseado en numerosos lugares en su libro sin que [estos errores] puedan ser excusables. Tampoco existe forma de librarlo de [adscribirle estos errores] pues su procedimiento y su demostración están construidos sobre su fantasía.

[§12] Cuando vimos esto, nos invitó [B. fol. 2v] todo lo que hemos mencionado a la composición de este libro. Lo hicimos preceder de una introducción inspirada en el libro de Teodosio que pueda facilitar su comprensión y su manejo. Lo dotamos de demostraciones para que el libro fuera autosuficiente y no necesitara más referencias externas que el libro de Euclides, pues no hay otra opción. A ello sumamos los contenidos introductorios que nos pareció y lo que creímos conveniente de la introducción de su libro. Separamos todo esto y lo dispusimos como [Es ${ }^{1}$ fol. $3 \mathrm{v}$ ] primer libro para que sirviera de aproximación y facilitara su lectura al estudiante y para que se detuviera en él [únicamente] una semana aproximadamente. Separamos la parte teórica de la parte práctica, la resumimos y las dispusimos por separado pues la única parte útil actualmente del Libro del Almagesto es sólo la teórica, dado que la parte práctica ya no es útil por numerosas causas. Por esta razón los astrónomos posteriores han separado la parte práctica en los ziŷes, lo que facilita y aproxima el uso. [Es ${ }^{2}$ fol. 
3r] Extendimos y comentamos en la medida de nuestra capacidad los temas que había abreviado en exceso. Introdujimos demostraciones en aquellos enunciados que [Ptolomeo] no demostró y que asumió como premisas al no poder demostrarlos.

[§13] Dado que la búsqueda de la verdad, preferirla y divulgarla es una obligación y que no hay que temer a quien se ha apartado de ella aunque sea alguien importante, ni hay que despreciar a quien la haya encontrado aunque sea alguien sin importancia - y nos inspiramos en lo que hizo Aristóteles, al refutar a su maestro Platón pues dijo excusándose: «Cuando la verdad y Platón discuten, si bien ambos me son queridos, la verdad me es más querida»— consideramos que sería conveniente enumerar en este momento los puntos en los que este hombre fantaseó y mencionar los lugares de nuestro libro en los que aparecen para que los que ya han avanzado en esta ciencia puedan dirigirse a ellos con facilidad, detenerse en los mismos y los consideren con detenimiento. Si nuestro parecer [sobre estos puntos] es verdadero, se adquirirá ciencia y nosotros recompensa; y si es erróneo, debe vencer la verdad, se nos recusará y se nos salvará de la oscuridad de la ignorancia, adquiriremos ciencia y se adquirirá recompensa y favor. Y Dios, sea ensalzado, asiste a la rectitud con $\mathrm{Su}$ misericordia.

[§14] [Lista de errores]

- [1] [Ptolomeo] cometió un error en el segundo capítulo del libro cuarto del Almagesto sobre los períodos de los ciclos de la Luna.

- [2] Cometió otro error en el capítulo décimo del libro quinto del Almagesto. La mención de ambos errores aparece en el cuarto libro de esta obra.

- [3] Hay otro error en los límites de los eclipses solares.

- [4] Hay otro error en el cálculo de los eclipses lunares.

- [5] Hay otro error en el cálculo de los eclipses solares y en los valores de sus fases. Todo esto se menciona en el quinto libro de esta obra.

- [6] Hay otro error en el cálculo del eclipse solar en la delimitación de la paralaje de la Luna en latitud donde lo añade a la [longitud] eclíptica cuando debería haberlo añadido a la propia Luna. Sin embargo esto no lo mencionaremos en nuestro libro pues sólo es necesario para la elaboración de tablas que se emplean en el cálculo del eclipse solar, y esto pertenece al ámbito de las cuestiones prácticas.

Al-Qanțara (AQ) XXX 1, enero-junio 2009, pp. 83-136 ISSN 0211-3589 
- [7] Hay otro error en el comienzo de libro noveno del Almagesto al considerar que las esferas de Venus y Mercurio se encuentran debajo del Sol, lo que se deduce de sus apreciaciones sobre la paralaje del sol. Sin embargo ambos planetas no tienen una paralaje sensible. De la relación que menciona del radio de sus dos [Es ${ }^{2}$ fol. $\left.3 \mathrm{v}\right]$ epiciclos con el radio de su deferente y de sus afirmaciones en torno a las cuestiones de las hipótesis para sus latitudes, se desprende que es obligado que estén por encima del Sol.

- [8] Hay otro error en sus palabras cuando afirma que estos dos [planetas] no pasan por las líneas tendidas [B. fol. 3r] entre el Sol y nuestra vista.

- [9] Se encuentra otro error en los apogeos de Venus y Mercurio, pues aplica la reciprocidad al tercer y cuarto teorema del capítulo sexto del libro noveno del Almagesto. Sin embargo en ambos casos no puede aplicarse la reciprocidad, pues no comprendió lo que querían decir los antiguos con las elongaciones contrarias en los planetas Venus y Mercurio ${ }^{107}$.

- [10] Existe otro error en que [Ptolomeo] consideró que los segmentos KE y TE ${ }^{108}$ de esta figura ${ }^{109}$ son el radio del deferente de Mercurio, lo cual no es así. Todo esto se encuentra en el libro séptimo de esta obra.

- [11] Existe otro error en la obtención [Es ${ }^{1}$ fol. 4r] de los dos puntos de las estaciones (wuqüf) de los planetas.

- [12] Existe otro error en que considera que la variación de los arcos de retrogradación de los planetas depende de la variación de las distancias entre el centro de los epiciclos y el centro de la esfera de las estrellas fijas. Sin embargo, este tema no es así.

- [13] Existe otro error en que considera que las dos estaciones (maqāmayn) son iguales de forma absoluta, pero esto no es así.

\footnotetext{
107 Para determinar el apogeo del planeta es necesario que las elongaciones máximas y opuestas del planeta al Sol medio cumplan condiciones contrarias con lo que estas elongaciones serán necesariamente simétricas respecto a la línea de los ápsides del deferente. Por ejemplo, para que dos elongaciones sean simétricas respecto al eje de los ápsides, si la elongación matutina crece rápidamente, la elongación vespertina debe decrecer también rápidamente. Cf. Samsó, "Ibn al-Haytham", 199-225, especialmente 216-221.

${ }_{108}$ Cf. PtA, 447, fig. 9.4. De hecho, Ŷābir b. Aflah se equivoca en el segundo segmento pues, pese a aludir al segmento TE, según el contenido de la crítica en el Libro VIII se deduce que se refiere al segmento TD, o bien $\Theta D$ según la transcripción de Toomer. Cf. Samsó, "Ibn al-Haytham", 215.

109 El Ms. B. 3r añade "del cuarto teorema de este capítulo".
}

Al-Qanțara (AQ) XXX 1, enero-junio 2009, pp. 83-136 ISSN 0211-3589 
- [14] Existe otro error en la delimitación del momento de estación de un astro y en el valor del tiempo de su retrogradación hasta el punto de que este error en el caso de Marte, en cuanto a la estimación del período de su retrogradación, puede llegar a ser de 18 días y en el caso de Venus de unos dos días y medio. Estos son tiempos excesivos. En la mayor parte de los casos el error para estos dos planetas es perceptible a los sentidos. En cuanto al resto de los planetas la medida del error no es perceptible, por razones que no hay que mencionar aquí. Todo esto se encuentra en el libro octavo de nuestra obra.

- [15] Existe un error en el teorema once del libro trece del Almagesto que coincide con el teorema cuarto del libro noveno de esta obra.

[§15] Hemos corregido todos los errores que hemos señalado en los diversos lugares mencionados de nuestra obra. Pedimos a Dios, sea ensalzado, la protección contra el error y el desliz así como la guía hacia la rectitud en toda palabra y obra por gracia Suya, quien no tiene asociado [en Su unidad]. Y ha llegado el momento de que comencemos con lo necesario para su introducción. Dios es Quien concede la ayuda. Que nuestro señor Muhammad y toda su familia reciban la bendición de Dios.

\section{Edición}

$$
\begin{aligned}
& \text { [Es }{ }^{1} \text { fol. 2v, Es }{ }^{2} \text { fol. 1v y B. fol. 1v] } \\
& \text { [1\$] بسم الله الرحمان الرحيم }
\end{aligned}
$$

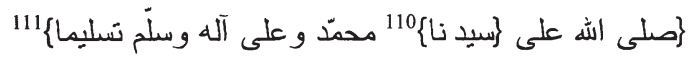

110 No aparece en Ms. Es².

111 No aparece en Ms. B.

Al-Qanțara (AQ) XXX 1, enero-junio 2009, pp. 83-136 ISSN 0211-3589 


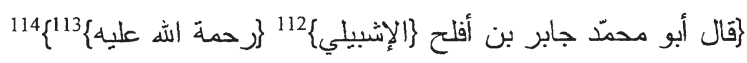

الحمد لله الأول بلا ابتداء والأخر بلا انتهاء اللذي خلق الأشياء على غير نظير وقدّر ها

بحكمته أحسن تقدير وصلّى الله على محمد نبيه البشير وسر اجه المنير الذي هدى به الخلائق

$$
\text { و أبان به الحقائق. }
$$

[2§] وبعد فإن الله تعالى شرتّ نوع الإنسان وفضلّه على جميع [أنو اع\{115 الحيوان

بالعقل الذي جعله نورا يستضئ به \}في\{16 الظلم ويتعلْم به ما لم يكن يعلم. فقال كجلّ جلهه\{17

هوخخلق الإنسان علّمه البيانهيه.118 وخاطب تبارك اسمه محمًّا عبده ورسوله صلوات اله عليه

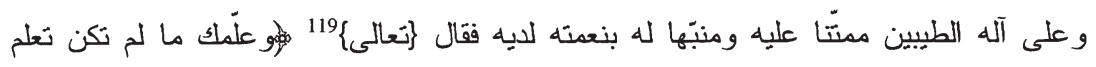

وكان فضل اله عليك عظيماهُ.

\footnotetext{
${ }^{112}$ No aparece en Ms. B.

${ }^{113}$ No aparece en Ms. Es ${ }^{1}$.

${ }^{114}$ No aparece en Ms. Es ${ }^{2}$.

${ }^{115}$ No aparece en Ms. Es ${ }^{1}$.

${ }^{116}$ Ms. B. “من".

${ }^{117}$ Ms. B. "نعالى".

${ }^{118}$ Corán 55,4 .

${ }^{119}$ No aparece en los Mss. Es ${ }^{1}, \mathrm{Es}^{2}$.

${ }^{120}$ Corán 4, 113.
}

Al-Qanțara (AQ) XXX 1, enero-junio 2009, pp. 83-136 ISSN 0211-3589 


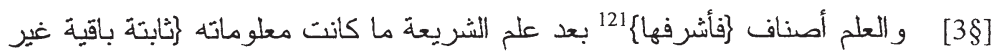

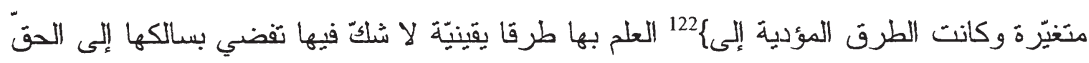
اليقين.

[4§] وعلم الهيئة \}بحركات\{123 الشمس و القمر و النجوم ومعرفة أفلاكها وما يلزم عن

ذلك علم يفضل كثير ا من العلوم لاجتماع وجوه الفضل فيه، فإنّ معلوماته ثابتَّة باقية غير متغيرة

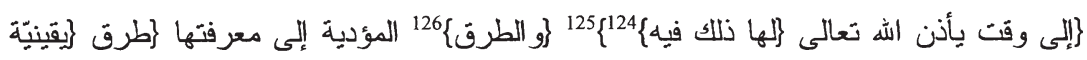
بيّنة \{27. فحصل لله الفضل من وجو هلج. 128

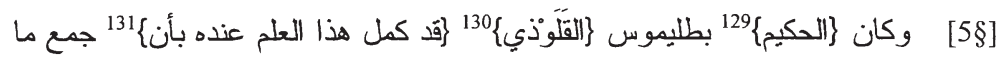
أدركه القدماء قبله وأضاف \}[إليه\{132 ما أدركه هو بعدهم. وسطر جميع ذلك في كتابه

${ }^{121}$ Ms. Es "فأفضلها", si bien corregido en el margen "فأُشرفها".

${ }^{122}$ Aparece borrado en Ms. B., excepto la palabra " "خابتَ" parcialmente borrada.

${ }^{123}$ Ms. Es "2لدركت".

${ }^{124}$ No aparece en Ms. B.

${ }^{125}$ Ms. Es "،لإلى الوقت الذي شاء الله بذلك فيه".

${ }^{126}$ Ms. Es “والطريق".

${ }^{127}$ Ms. B. "بيتنة يقينية".

${ }^{128}$ Ms. Es: طرق يقينية والمقصود من الاطلاع عليه الوقوف على عجانب من حكمة اله يتحيَر فيها" الألباب وتقضي نفوس العارفين منها العجب العجاب محصل له الفضل من وجوهه"

${ }^{129}$ No aparece en los Mss. Es ${ }^{1}$, Es ${ }^{2}$. 
المعروف\{133 بالمجسطي. فكان بذلك رب النعمة الجزيلة علينا فو النعمة الجسيمة\{134 لدينا.

وصار كتابه $\} ف ي\left\{135\right.$ ذلك ${ }^{135}$ : يصعب\{138 على الناظر فيه لمعان شتّى

- [5.1] منها أنّه جامع للعلم و العمل. فاضطر" من طريق العمل إلى ضرب الأعداد

[Es ${ }^{2}$ fol. 2r] بعضها في بعض\{139 وقسمة بعضها على بعض وتجذيرها وتصنيف\}

الجداول المنصرّفة في العمل. فطال الكتاب \}لذلك\{140 وتقستم العلم فيه $\}$ وأمزج\{141 بالعمل

$$
\text { امتز اجا يعسر \}به } 142 \text { على قارئه } 143 .
$$

${ }^{130}$ Forma arabizada de Claudio Ptolomeo. No aparece en Ms. Es ${ }^{2}$.

${ }^{131}$ No aparece en los Mss. Es ${ }^{1}$, B.

${ }^{132}$ No aparece en Ms. B.

${ }^{133}$ Ms. Es" "المسمَى".

${ }^{134}$ Mss. B. , Es "و المنّة الجسميّة"

${ }^{135}$ No aparece en los Mss. Es ${ }^{1}$, B.

${ }^{136}$ No aparece en Ms. B.

${ }^{137}$ Ms. B. “ماني هذا العلم".

138 Ms. B. "صعب!".

${ }^{139}$ Ms. Es "في الأعداد".

${ }^{140}$ Ms. Es “بذلك".

${ }^{141}$ Ms. B. "وامتزج". A esta altura, fragmento ilegible de media línea en Ms. Es².

${ }^{142}$ No aparece en Ms. Es ${ }^{2}$. 
- [5.2§]ومنها أنّه استعمل في \}كثير من\{144 بر اهينه الشكل القطتّاع وهو شكل (صعب

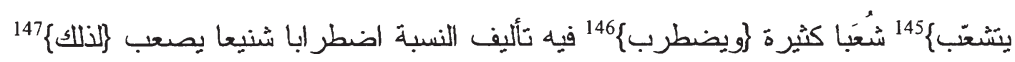
على الناظر (فيه\{148 ضبطه وحصره و إنتاج ما [ينتج]149 منه. - [5.3 [منها أيضا أنّه أحال في بر اهينه على كتاب (تاودسيوس\{150 وعلى كتاب ميلاوش\{151 وهما صعبان عويصان. ليس يتهيًأ للطالب المجتهد الوقوف عليهما والتكربب فِيهما وفي الشكل القطّاع في أقلّ من حول (كامل\{152. فربّما كسل بعد ذلك أو قطع به الزمان عن الشروع في الكتاب.

${ }^{143}$ Sigue aproximadamente media línea ilegible en $\mathrm{Ms} . \mathrm{Es}^{2}$ que no aparece en el resto de los manuscritos.

${ }^{144}$ No aparece en Ms. Es ${ }^{2}$.

${ }^{145}$ Ms. Es “"يصعب ويشعب".

${ }^{146}$ Ms. Es “وتضطرب".

${ }^{147}$ Ms. B. “بذّلك". No aparece en Ms. Es².

${ }^{148}$ Interlineado en Ms. Es², seguido de "للك".

${ }^{149}$ Ms. B. “ينتجه".

${ }^{150}$ Ms. B. “ثاودسيوس”.

${ }^{151}$ Ms. B. "ملاناوس".

${ }^{152}$ No aparece en Ms. B. 


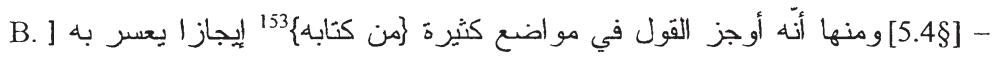
[fol. 2r

$$
\text { الكسل \}و إفر اط الملل\{156. }
$$

- [5.5\$]ومنها أنّه بتداول المترجمين له من لسان إلى لسان عرّض فيه من التقديم و التأخير في العبارة و الفصل بين معانيه، ما بضلَل القارئ له ويحيّره. ويوقفه (في موضع

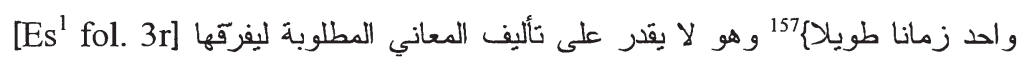

$$
\text { \}بعضها عن بعض\{158. فربّما قطع \}بها\{159 ذلك عن التمادي في النظر. }
$$

[6§] و اتَقق لنا نحن من الولوع بهذا العلم و المحبّة لله [للأمورج160 التي ذكرناها ولما

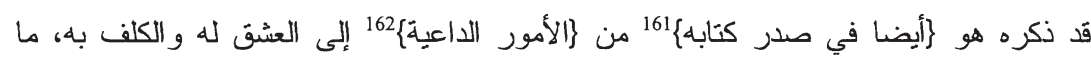

${ }^{153}$ Ms. B. “منه".

${ }^{154}$ Mss. B., Es " "يَحتير”".

${ }^{155}$ Ms. Es "فيه".

${ }^{156}$ No aparece en Ms. B.

${ }^{157}$ No aparece en Ms. B.

${ }^{158}$ No aparece en Ms. B.

${ }^{159}$ Ms. B. , Es "“ب̣".

${ }^{160}$ Ms. B. “"من أجل الأمور".

${ }^{161}$ Ms. Es "في صدر كتابه أيضان".

${ }^{162}$ No aparece en Ms. B.

Al-Qanțara (AQ) XXX 1, enero-junio 2009, pp. 83-136 ISSN 0211-3589 
حملنا على \}إمعان\{163 النظر فيه و احتمال المشقة و الصعوبة العارضة لقارئه فو استعينا على ذلك بما كان قد تقدّم لنا من التمرّن في صناعة الهندسة و الاطمئنان 164 بهاج\{165 إلى أن حصل لنا بحمد الله \}تبارك وتعالى فهم\{166 جميع ما تضمّنه هذا الكتاب من علم الهيئة. ولم نزل بعد ذلك نُيم النظر ونو الي البحث و التفكر فيما يمكن أن يسهل به \}(مر امج167 هذا العلم الجسيم كويقرتب\{168 به فهم هذا الكتاب العظيم\{169.

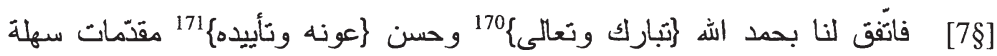

وجيزة ليستغني\{172 بها عن كتاب ميلاوش وعن الشكل القطّاع وعن \}أكثر كتاب\{173 تاودسيوس،

${ }^{163}$ No aparece en Ms. Es².

${ }^{164}$ Palabra de lectura dudosa en el texto; probablemente se trate de "iتنان||". Agradezco al Prof. Samsó la sugerencia de "اطمئنان" basada en la confusión habitual en ámbito andalusí de la $t$ por $t$.

${ }^{165}$ No aparece en los Mss. Es ${ }^{1}, \mathrm{~B}$.

${ }^{166}$ No aparece en Ms. B. Ms. Es "فه" 2 ".

${ }^{167}$ Ms. B. “من أمر". Ilegible en Ms. Es².

${ }^{168}$ Ms. B. “ويعرف”.

${ }^{169}$ Ms. Es “هذا الكتاب ويقرب مر ام هذا العلم الجسيح”.

${ }^{170}$ No aparece en los Mss. B., Es².

${ }^{171}$ Mss. B., Es "تأييده".

${ }^{172}$ Ms. B. "ليستغني".

${ }^{173}$ Ms. B. “أكر". Ms. Es" "كثرب".

Al-Qanțara (AQ) XXX 1, enero-junio 2009, pp. 83-136 ISSN 0211-3589 


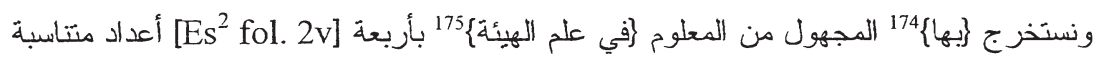

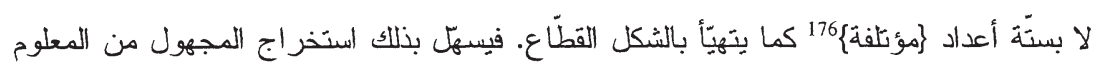
إذ كان احتياجنا فيه إلى معلومات أقلّ. وصار لذلك ادر اكه سهل المر ام قليل الاشتباك والآتحام.

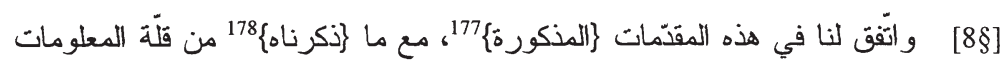

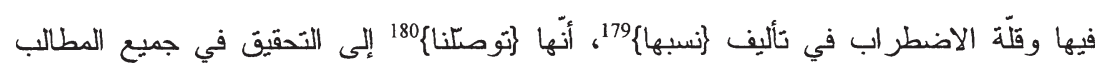
التي تستعمل فيها. ولا (نحتاج\{181 معها إلى استعمال الخطوط المستقيمة وزو اياها مكان القسي وزو اياها كما فعل \}بطليموس\{182 في مواضع من كتابه، إذ لم يمكنه سوى ذلك. ويدخل ذلك من التقريب ما لا خفاء به.

${ }^{174}$ Ms. B. "به".

${ }^{175}$ No aparece en los Mss. Es ${ }^{1}$, B.

${ }^{176}$ Ms. B. “متافة".

${ }^{177}$ Ms. B. "مذكور ات"

${ }^{178}$ Ms. B. “ذكرنا".

${ }^{179}$ Ms. Es "نسبتها".

${ }^{180}$ Mss. Es", Es" "توصتان"

${ }^{181}$ Ms. Es “تحياج".

${ }^{182}$ Mss. B., Es “بطلميوس”.

Al-Qanțara (AQ) XXX 1, enero-junio 2009, pp. 83-136 ISSN 0211-3589 
[98]

ثثانى حلق. و اتَّقَ لنا آلة واحدة ليس تحتاج فِيها إلا إلى حلقة واحدة وربع أخرى ومسطرة

$$
\text { وتغني عن جميع الآلات التي ذكرها\{185. }
$$

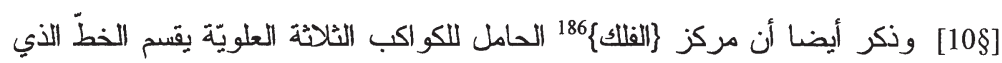

بين مركز الحركة المسنتوية وبين مركز \}فلك البروج\{187 بنصفين. وجعل ذلك من الأمور التي

تؤخذ مُسلَّمة، إذ لم يمكنه \}طريق يصل به إلى معرفة ذلك\{188 ببرهان. و اتَفق لنا بحمد (اله

$$
\text { تبارك وتعالى طريق\{189 نصل به إلى \}معرفة\{190 نللك ببر هان (صحيح\{191. }
$$

[11] ولمّا أمعنّا النظر، ظهر لنا أنه قد لحقه ما يلحق البشر من النقصان الخاصن

بطبيعة الإنسان $\}$ ببحان\{192 المنفرد بالكمال نعالى اسمه. وذلك أنته مع ما \}ذكرناه\{193 آنفا من

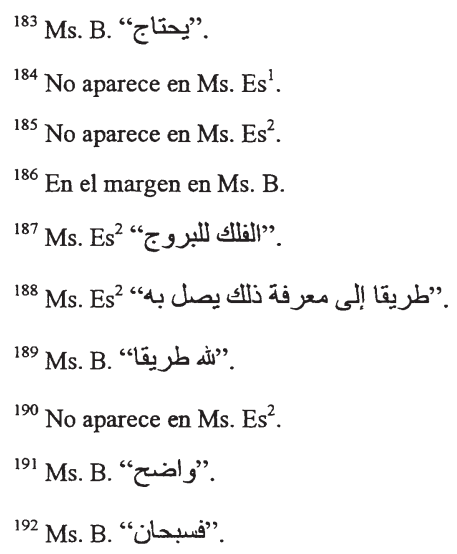


تقصيره، وجدناه قد وهم في مواضع كثيرة من كتابه وممّا لا بسع العذر عنه فيها. و لا يوجد له سبيل إلىى التخلّص \}منها لأنّ عمله وبر هانه ذلك\{194 مبني على وهمه. [128] ولمتا رأينا نلك، دعانا [B. fol. 2v] جميع ما ذكرناه إلى تأليف هذا الكتاب. فقدمنا من كتاب \}تاودسيوس\{195 ما يقرب فهمه ويسهل مأخذه. وأتينا كبالبر اهين\{196 عليه كي

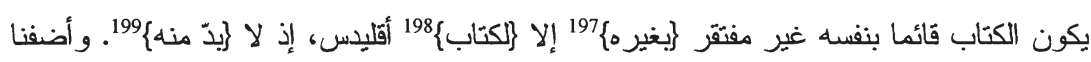
إلىى \}ذلك\{200 المقدّمات التي ظهرت لنا وما رأينا تقديمه من كتابه. وأفردنا لجميع ذلك مقالة وجعلناها [Es fol. 3v] أول مقالاته وهي من القرب و السهولة \}ما\{201 يمكن الناظر فيها أن يقر أها كويقف عليها\{202 في جمعة أو نحو ها. وجرّدنا العلم فيه من العمل ولخّصناه وجمعنا بعض

${ }^{193}$ Ms. B. "نكرنا".

${ }^{194}$ Ms. B. “"من الذي و هم فينه وذلك أنت بر هانه و علمه". Ms. Es "منها لأن بر هانه و عمله على ذلكي".

${ }^{195}$ Ms. B. “ثاودسيوس”.

${ }^{196}$ Ms. Es "بالبر هان".

${ }^{197}$ No aparece en los Mss. Es ${ }^{1}, \mathrm{Es}^{2}$.

${ }^{198}$ Mss. B., Es “"إلى كتاب".

${ }^{199}$ Mss. Es ${ }^{1}$, Es " “ندوحة عند".

${ }^{200}$ Ms. B. "كلt"l.

${ }^{201}$ Ms. Es “بحيث".

${ }^{202}$ No aparece en Ms. Es ${ }^{2}$

Al-Qanțara (AQ) XXX 1, enero-junio 2009, pp. 83-136 ISSN 0211-3589 
إلى بعض \}إذج\{203 الفائدة من كتاب المجسطي الآن إنّما هو العلم فقط لأنّ العمل به الآن لا يمكن لأسباب كثيرة. ولذلك جرّد المتأخرون العمل منه في الزيجات فصار أسهل وأقرب تتاو لا. Es² ] و بسطنا وشرحنا المو اضع التي أوجز القول فيها بقدر ما أمكننا. و أتينا بالبر اهين على ما 3rol. 3r ذكره ذكر ا مرسلا ولم يبرهن عليه و على ما أخذه مُسلَّا إذلم لمكنه البرهان عليه.

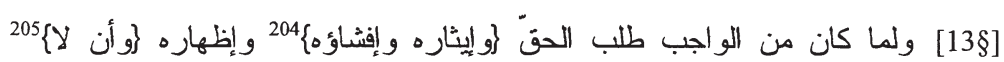
\}يهاب\{206 من نكب عنه وإن كبر \}و لا يستهان بمن وجده وإن صغر\{207 - وكان لنا أسوة فيما فعله أرسطوطاليس إذ قصد الردّ على شيخه أفلاطون فقال معنذر ا "تخاصم الحق" وأفلاطون

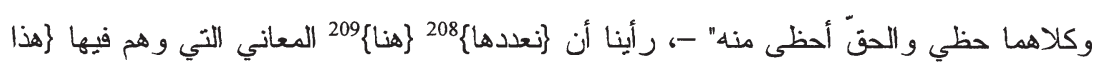
الرجل\{210 ونذكر مواضعها من \}كتابنا هذا\{211 كي يقصد إليها بسهولة من أر اد الوقوف عليها

${ }^{203}$ Ms. B. “ذإ!””.

${ }^{204}$ No aparece en Ms. B.

${ }^{205} \mathrm{Ms} . \mathrm{Es}^{2}$ "yأو".

${ }^{206}$ Ms. B. "يحاب".

${ }^{207}$ No aparece en Ms. B.

${ }^{208}$ Ms. Es " "نذكر ها".

${ }^{209}$ No aparece en los Mss. B., Es ${ }^{2}$.

${ }^{210}$ No aparece en Ms. B.

${ }^{211} \mathrm{Ms}$. Es "كتابه”.

Al-Qanțara (AQ) XXX 1, enero-junio 2009, pp. 83-136 ISSN 0211-3589 


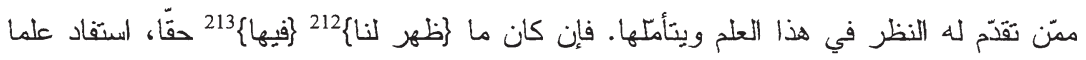
و استقفنا أجرا؛ وإن كان باطلا، فواجب عليه أن ينصر الحق" ويردّ (علينا فيستقذنا\{214من من \}ظلمة 215 الجهل ويفيدنا علما ويستفيد أجر ا وشكر ا. و اله \}تعالى يوفق للصو اب برحمنه\{16. [14§] [ [اتمة الأخطاء]

- [1] فمن ذلك أنها وهم في النوع الثاني من المقالة الرابعة من كتابه في أزمان القمر الدوريّة.

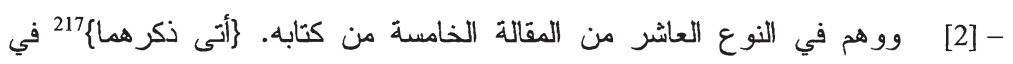
المقالة الر ابعة من كتابنا هذا. - [3] و هم \}أيضا\{218 في حدود الكسوفات الشمسيّة.

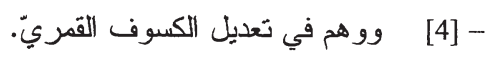
${ }^{212}$ Ms. B. "نكرناه".

${ }^{213}$ No aparece en Ms. Es ${ }^{2}$.

${ }^{214}$ Ms. Es "على قائله فنستفده".

${ }^{215}$ Ms. B. “جَجَّ".

${ }^{216}$ Ms. Es " "الموفق للصواب”.

${ }^{217}$ Ms. B. “وأنا أذكر هماب".

${ }^{218}$ No aparece en los Mss. Es ${ }^{1}, \mathrm{Es}^{2}$.

Al-Qanțara (AQ) XXX 1, enero-junio 2009, pp. 83-136 ISSN 0211-3589 
- [5] ووهم في تعديل الكسوف الثمسيّ وفي مقادير أزمنته وجميع ذلك مذكور في المقالة الخامسة من كتابنا هذا.

- [6] ووهم أيضا في تعديل الكسوف الشمسيّ في تحديد اختلاف منظر القمر في العرض بأن أضاف ذلك إلى فلك البروج و إنما كان يجب أن يضيفه إلى القهر نفسه. وهذا

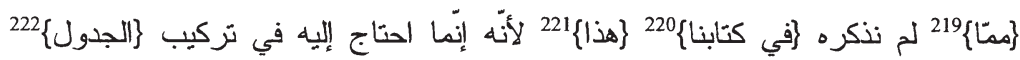
الذي يعدل به الكسوف الشمسيّ فو هذا\{223من الأمور العمليّة.

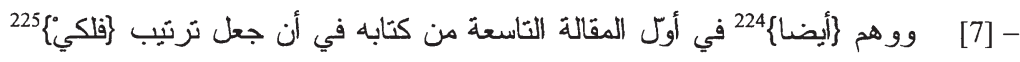
الزهرة و عطارد تحت فلك الشمس والذي \}\}يخرج ممأ226 ذكره من اختلاف منظر

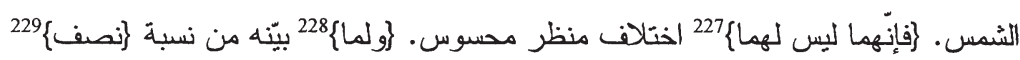

\footnotetext{
${ }^{219}$ No aparece en Ms. Es ${ }^{2}$.

${ }^{220}$ No aparece en Ms. B.

${ }^{221}$ No aparece en los Mss. Es ${ }^{1}$, B.

222 Ms. B. "جداول".

${ }^{223} \mathrm{Ms}$. Es "وهو" هو

${ }^{224}$ No aparece en los Mss. Es ${ }^{1}, \mathrm{Es}^{2}$.

${ }^{225}$ Ms. Es “" “纤”.

${ }^{226}$ En el margen en Ms. Es ${ }^{2}$.

${ }^{227}$ Ms. Es "و إنها ليس لها".

${ }^{228}$ Ms. Es "وممَ".
}

Al-Qanțara (AQ) XXX 1, enero-junio 2009, pp. 83-136 ISSN 0211-3589 
قطر فلكي [Es fol. 3v تدوير هما إلى نصف قطر الفلك الحامل لهما وممّا ذكره في أمر الأصول الموضو عة لعروضهمائ230، إنهما فوق \}فلك\{231 الشمس ضرورة.

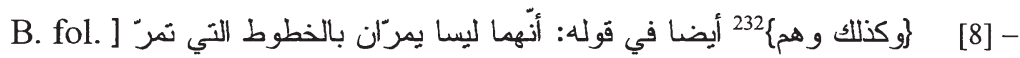
3r

- [9] [وو هم في وجود البعد الأبعد للزهرة وعطارد، بأن عكس الشكل الثالث و الشكل الر ابع من النوع السادس من المقالة التاسعة من كتابه\{233. كو هو\{234 ممّا لا ينعكس، وبأنته لم يفهم ما أر اده القدماء بالأبعاد المتضادّة [لكوكبي الزهرة]235 و عطارد.

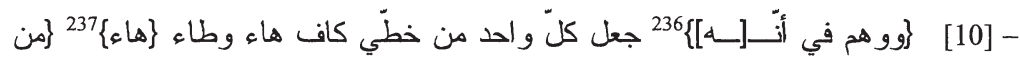
ذللك الشكل\{238 نصف قطر \}(الفلك\{239 الحامل لعطارد فوليس كذلك\{240. وجميع ذللك في المقالة السابعة من كتابنا هذا.

${ }^{229}$ No aparece en Ms. Es ${ }^{2}$.

${ }^{230}$ Ms. B. "“بعطيه أصوله".

${ }^{231}$ No aparece en los Mss. Es ${ }^{1}, \mathrm{Es}^{2}$.

${ }^{232}$ Ms. B. “ووهم".

${ }^{233}$ Ms. Es 2 ووهم في الشكل الرابع من النوع السادس من المقالة التاسعة من كتابه في أن عكس بر هان وره" "ذلك الشكل"

${ }^{234}$ Ms. B. "وهما".

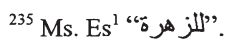

Al-Qanțara (AQ) XXX 1, enero-junio 2009, pp. 83-136 ISSN 0211-3589 
- [11] وو هم في استخر اج [Es fol. fr] نقطتي الوقوف للكوكب من الكو اكب السيارة. - [12 ووهم في أن جعل تفاضل قسي الرجوع للكوكب بحسب تفاضل أبعاد مركز فلكى\{241 التدوير من مركز فلك البروج وليس الأمر كنلك. - [13 [ [وو هم بأن جعل المقامين للكوكب متساويين على الإطلاق \}وليسا\{242 كذللك\{243. - [14] وو هم في تحديد وقَت الوقوف للكوكب وفي مقدار زمان رجوعه، حتى أنه بِكن أن يدخل ذلك الوهم في \}زمان رجوع\{244 كوكب المريخ وحده \}في\{245 مقدار زمان رجو عها من الخطاء\{246 نحو ثمانية عشر يوما وفي \}زمان رجوع\{247 كوكب الز هرة نحو يومين ونصف، و إنّما يتّقتق ذلك في الفرط من الزمان. و أمّا (في أكثر الأمر\{248 فالخطاء

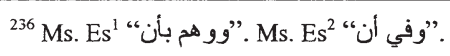

${ }^{237}$ Ms. Es " “ب”.

${ }^{238}$ Ms. B. “من الثكل الرابع من ذلك النوع".

${ }^{239}$ No aparece en los Mss. Es ${ }^{1}, \mathrm{Es}^{2}$.

${ }^{240}$ No aparece en Ms. Es ${ }^{2}$.

${ }^{241}$ No aparece en Ms. B.

${ }^{242}$ Mss. B., Es "وليس".

${ }^{243}$ En el margen en Ms. Es ${ }^{2}$.

${ }^{244}$ No aparece en los Mss. Es ${ }^{1}, \mathrm{Es}^{2}$.

${ }^{245}$ Ms. Es " "وفي".

${ }^{246}$ No aparece en Ms. B.

${ }^{247}$ No aparece en los Mss. Es ${ }^{1}, \mathrm{Es}^{2}$.

${ }^{248}$ Ms. B. “عثى الأكثر”. 
في \}زمان رجوع\{249 هذين الكوكبين ظاهر بيّن للحس. و أمتا في غير هما فيخفى مقدار الخطاء (فيها\{250، لمعان ليس هذا موضع ذكرها. وجميع ذللك مذكور في المقالة الثامنة من كتابنا هذا. - [15] ووهم في الشكل الحادي عشر هن المقالة النالثة في الشكل الرابع من المقالة التاسعة من كتابنا هذا. [15\$] وقد أصلحنا جميع ما ذكرناه ممّا وهم فيه في \}المو اضع\{253 التي ذكرناها كن

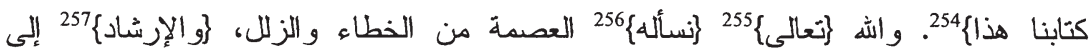

${ }^{249}$ No aparece en los Mss. Es ${ }^{1}, \mathrm{Es}^{2}$.

${ }^{250}$ Ms. Es "فيهما".

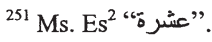

${ }^{252}$ En el margen en Ms. Es ${ }^{2}$.

${ }^{253}$ Ms. B. "موضعه".

${ }^{254}$ No aparece en Ms. B.

${ }^{255}$ No aparece en Ms. Es ${ }^{2}$.

${ }^{256}$ Mss. B., Es "أسأله".

${ }^{257}$ Ms. Es “ونسأله الإرشاد”.

Al-Qanțara (AQ) XXX 1, enero-junio 2009, pp. 83-136 ISSN 0211-3589 


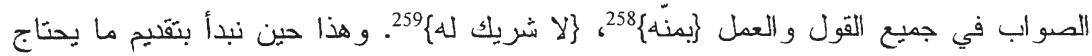

$$
\begin{aligned}
& \text { \}إلى ثقديمه\{260. كو الله المستعان على ذلك وصلّى الله على سيّدنا محمد و آله أجمعين\{261. }
\end{aligned}
$$

Recibido: $22 / 10 / 2007$

Aceptado: 05/06/2008

${ }^{258}$ Ms. B. "بمنهل وحوله وهو حسبنا ونتم الوكيل".

${ }^{259}$ No aparece en Ms. B. Ms. Es" “لا ربّ غيره ولا شريك له”.

${ }^{260}$ Ms. Es "“إليه".

${ }^{261}$ No aparece en los Mss. Es ${ }^{1}$, Es$^{2}$.

Al-Qanțara (AQ) XXX 1, enero-junio 2009, pp. 83-136 ISSN 0211-3589 